ESAIM: COCV 19 (2013) 255-273

DOI: $10.1051 / \mathrm{cocv} / 2012008$
ESAIM: Control, Optimisation and Calculus of Variations

www.esaim-cocv.org

\title{
A LOWER BOUND ON LOCAL ENERGY OF PARTIAL SUM OF EIGENFUNCTIONS FOR LAPLACE-BELTRAMI OPERATORS *
}

\author{
QI L $\ddot{U}^{1,2}$
}

\begin{abstract}
In this paper, a lower bound is established for the local energy of partial sum of eigenfunctions for Laplace-Beltrami operators (in Riemannian manifolds with low regularity data) with general boundary condition. This result is a consequence of a new pointwise and weighted estimate for Laplace-Beltrami operators, a construction of some nonnegative function with arbitrary given critical point location in the manifold, and also two interpolation results for solutions of elliptic equations with lateral Robin boundary conditions.
\end{abstract}

Mathematics Subject Classification. 93B07.

Received October 28, 2011. Revised February 2, 2012.

Published online 11 May 2012.

\section{INTRODUCTION AND MAIN RESULT}

Let $M$ be a $d(d \in \mathbb{N})$ dimensional connected compact $C^{1}$-smooth Riemannian manifold with an $C^{2}$-smooth boundary $\Gamma$, and $\omega$ a nonempty open subset of $M$. Denote by $g$ the $C^{1}$-smooth Riemannian metric tensor on $M$; by $\mathcal{D}_{g}$ the Levi-Civita connection on $M$ induced by $g$; by $\nabla_{M}$, $\operatorname{div}_{M}$ and $\Delta_{M}$ the gradient operator, the divergence operator and the Laplace-Beltrami operator (on $M$ ) given by $\mathcal{D}_{g}$, respectively; by $(\cdot, \cdot)_{g}$ and $|\cdot|_{g}$ the inner product and the norm for the tangent vector of $M$ with respect to $g$, respectively; by $d_{g} x$ the volume element of $M$ with respect to $g$; and by $d_{g} \Gamma$ the volume element of $\Gamma$ induced by $g$. We refer to [3] for more details on the notation/tool used in this paper, say Sobolev spaces on Riemannian manifold. Fix any $T>0$, and put $Q=(0, T) \times M$ and $\Sigma=(0, T) \times \Gamma$. Throughout this paper, we use $C=C(M, \omega, d, g, T)$ to denote a generic positive constant, which may change from one place to another.

We define an unbounded operator $A$ on $L^{2}(M)$ by

$$
\left\{\begin{array}{l}
D(A)=\left\{u \in H^{2}(M): \widetilde{l} \frac{\partial_{M} u}{\partial \nu}+l u=0 \text { on } \Gamma\right\}, \\
A u=-\Delta_{M} u, \quad \forall u \in D(A),
\end{array}\right.
$$

Keywords and phrases. Lower bound, local energy, partial sum of eigenfunctions, Laplace-Beltrami operator, Robin boundary condition.

* This work is partially supported by the NSF of China under Grants 10831007, 11101070 and 60974035. This paper is an improved version of one chapter of the author's Ph.D. thesis [13] accomplished at Sichuan University under the guidance of Professor Xu Zhang. The author would like to take this opportunity to thank him deeply for his help.

1 School of Mathematical Sciences, University of Electronic Science and Technology of China, Chengdu 610054, P.R. China. luqi59@163.com

2 Basque Center for Applied Mathematics (BCAM), Mazarredo, 14, 48009 Bilbao Basque Country, Spain. 
where $\nu=\nu(x)$ is the unit outward normal vector of $M$ at $x \in \Gamma$ with respect to the metric $g,\left.\frac{\partial_{M} u}{\partial \nu}\right|_{\Gamma}=$ $\left.\left(\nabla_{M} u, \nu\right)_{g}\right|_{\Gamma}$, both $\tilde{l}$ and $l$ belong to $L^{\infty}(\Gamma)$ and satisfy $\tilde{l}=1, l \geq 0$ or $\tilde{l}=0, l>0$. Let $\left\{\lambda_{i}\right\}_{i=1}^{\infty}$ be the eigenvalues of $A$, and $\left\{e_{i}\right\}_{i=1}^{\infty}$ the corresponding eigenfunctions satisfying $\left|e_{i}\right|_{L^{2}(M)}=1$. It is easy to show that $0 \leq \lambda_{1} \leq \lambda_{2} \leq \ldots$, and $\left\{e_{i}\right\}_{i=1}^{\infty}$ constitutes an orthonormal basis of $L^{2}(M)$.

One can find the following result from $[4,7,9]$.

Theorem 1.1. If both $\Gamma$ and $g$ are $C^{\infty}, \widetilde{l}=0$ and $l=1$, then it holds that

$$
\sum_{\lambda_{i} \leq r}\left|a_{i}\right|^{2} \leq C \mathrm{e}^{C \sqrt{r}} \int_{\omega}\left|\sum_{\lambda_{i} \leq r} a_{i} e_{i}(x)\right|^{2} d_{g} x,
$$

for every $r>0$ and every choice of the coefficients $\left\{a_{i}\right\}_{\lambda_{i} \leq r}$ with $a_{i} \in \mathbb{C}$.

This result provides a delicate lower bound estimate for the local energy of partial sum of eigenfunctions for Laplace-Beltrami operators (in $C^{\infty}$-smooth Riemannian manifolds) with Dirichlet boundary condition . As remarked in [22], the power $\frac{1}{2}$ in the above $\mathrm{e}^{C \sqrt{r}}$ is sharp. In terms of the control theory language, inequality (1.2) can be viewed as an observability estimate for partial sum of eigenfunctions for operator $A$. Besides its obviously independent interest, this inequality has many applications in control theory. In [7], by means of a time iteration approach, Lebeau and Robbiano used (1.2) to obtain null controllability of the heat equation with homogeneous Dirichlet boundary condition. In [9], inequality (1.2) was addressed by Lebeau and Zuazua, and via which null controllability of a linear system of thermoelasticity was analyzed. Further applications of this inequality to controllability problems can be found in [11,15,16,21]. On the other hand, in [19], Wang used (1.2) to establish an $L^{\infty}$-null controllability for the heat equation, and especially, via which he solved a long-standing open problem in control theory for infinite dimensional systems, i.e., the Bang-Bang principle for time optimal control problem for the heat equation with a locally distributed controller. His results was recently extended to fractional order parabolic equations, see [12].

We remark that, in Theorem 1.1, both $\Gamma$ and $g$ are assumed to be $C^{\infty}$-smooth. Escauriaza pointed out that the $C^{\infty}$-regularity for $\Gamma$ can be weakened to be $C^{2}$ but his proof was not published (see Rem. 1.1 in [11]). In this paper, we shall address the sharp result in this respect and, in particularly, consider a similar problem but with more general boundary conditions.

The main result of this paper can be stated as follows:

Theorem 1.2. The conclusion in Theorem 1.1 still holds when the additional assumptions on $\Gamma, g, \widetilde{l}$ and $l$ therein are dropped.

Noting that the time iteration method developed in [7] does not depend on the boundary condition. Therefore, using Theorem 1.2 and this method, it is easy to obtain the corresponding controllability/optimal control results for equations with Robin boundary condition. On the other hand, Theorem 1.2 can also be employed to prove the null/approximate controllability of forward stochastic heat equations [14], which is, to the best of the author's knowledge, the first controllability result for forward stochastic partial differential equations with control acts only on the drift term.

Theorem 1.2 needs much lower regularities for both $\Gamma$ and $g$ than Theorem 1.1. Furthermore, Theorem 1.2 is for general Robin type boundary condition while Theorem 1.1 addresses only the homogeneous Dirichlet boundary condition.

In $[4,7,9]$, the authors employed a local Carleman estimate to establish Theorem 1.1. The homogeneous Dirichlet boundary condition plays an important role in their proof. However, it seems to be quite difficult to prove Theorem 1.2 by using the same method. Instead, in this paper, we shall use a global (in space) Carleman estimate to overcome the difficulties introduced by the general boundary condition. On the other hand, it deserves to point out that, although a related global Carleman estimate was established in [2] addressing observability estimates for quite general parabolic equations, the approach therein does not seem to be able to 
provide the desired sharp estimate " $\mathrm{e}^{C \sqrt{r}}$ " in Theorem 1.2. Indeed, in order to prove Theorem 1.2, we need to derive first a new pointwise and weighted estimate for Laplace-Beltrami operators (see Sect. 2), and then to prove the existence of a nonnegative function with arbitrary given critical point location in manifold $M$ (see Sect. 3), and also to show some interpolation results for solutions of elliptic equations with lateral Robin boundary conditions in a cylinder (see Sects. 4 and 5 ).

It is considerably easier to prove Theorem 1.2 with $\widetilde{l}=0$ and $l>0$ than the case with $\widetilde{l}=1$ and $l \geq 0$. Noting that in both cases we can use the same method to obtain the desired inequalities. Therefore in the sequel we only prove Theorem 1.2 for the case that $\widetilde{l}=1$ and $l \geq 0$. The proof of this theorem will be given in Section 6. Note also that, even for the case of Dirichlet boundary condition, our method seems to be more elementary and also self-contained than that in $[4,7,9]$.

\section{A pointwise And Weighted estimate For LAplace-Beltrami operators}

In this section, we establish a pointwise weighted estimate for Laplace-Beltrami operators on a given Riemannian manifold, which will play a key role in the sequel.

Let $N$ be a $n$-dimensional $(n \in \mathbb{N})$ Riemannian manifold with a $C^{1}$-metric tensor $b$. The meaning of $(\cdot, \cdot)_{b}$, $|\cdot|_{b}, \nabla_{N}, \operatorname{div}_{N}, \Delta_{N}$ and so on can be understood similarly as mentioned at the very beginning of Section 1.

Let $H, H_{1}$ and $H_{2}$ be any given $C^{1}$-vector fields on $N$. We recall the following well-known formulas which will be useful later (e.g. [3], Chap. 1, [5], Chap. 3).

$$
\begin{aligned}
\operatorname{div}_{N}(h H) & =\left(\nabla_{N} h, H\right)_{b}+h \operatorname{div}_{N} H, \quad \forall h \in C^{1}(N), \\
\nabla_{N}\left(H_{1}, H_{2}\right)_{b} & =\left(\nabla_{N} H_{1}, H_{2}\right)_{b}+\left(\nabla_{N} H_{2}, H_{1}\right)_{b}+\left(\nabla_{N} b\right)\left(H_{1}, H_{2}\right),
\end{aligned}
$$

where $\left(\nabla_{N} H_{i}, H_{j}\right)_{b}$ stands for the contraction of the tensor $b \otimes \nabla_{N} H_{i} \otimes H_{j}(1 \leq i, j \leq 2, i+j=3),\left(\nabla_{N} b\right)\left(H_{1}, H_{2}\right)$ stands for the contraction of the tensor $\nabla_{N} b \otimes H_{1} \otimes H_{2}$. Also, for any $f \in C^{1}(N)$, we denote by $\nabla_{N}\left(\nabla_{N} f\right)$ the Hessian of $f$.

In the sequel, for arbitrary real function $\varphi \in C^{2}(N)$ and arbitrary positive real numbers $s$ and $\lambda$, we choose functions $\alpha$ and $\theta$ as follows:

$$
\alpha=\mathrm{e}^{\lambda \varphi}, \quad \theta=\mathrm{e}^{s \alpha} .
$$

We have the following result:

Theorem 2.1. Assume $v \in C^{2}(N)$ and put $w=\theta v$. Then it holds that

$$
\begin{aligned}
2 \theta^{2}\left|\Delta_{N} v\right|^{2}+D \geq & B_{1}\left|\nabla_{N} w\right|_{b}^{2}+B_{2} w^{2}+4 s \lambda^{2}\left(\nabla_{N}\left(\alpha\left|\nabla_{N} \varphi\right|_{b}^{2}\right), \nabla_{N} w\right)_{b} w \\
& +4 s \lambda^{2} \alpha\left(\nabla_{N} w, \nabla_{N} \varphi\right)_{b}^{2}+4 s \lambda \alpha\left(\nabla_{N} w,\left(\left[\nabla_{N}\left(\nabla_{N} \varphi\right)\right], \nabla_{N} w\right)_{b}\right)_{b} \\
& +4 s \lambda \alpha\left(\nabla_{N} w,\left(\nabla_{N} b\right)\left(\nabla_{N} w, \nabla_{N} \varphi\right)\right)_{b}-2 s \lambda \alpha\left(\left(\nabla_{N} b\right)\left(\nabla_{N} w, \nabla_{N} w\right), \nabla_{N} \varphi\right)_{b}
\end{aligned}
$$

where

$$
\left\{\begin{aligned}
D= & 2 s \lambda \operatorname{div}_{N}\left[2 \lambda \alpha\left|\nabla_{N} \varphi\right|_{b}^{2} w \nabla_{N} w+s^{2} \lambda^{2} \alpha^{3}\left|\nabla_{N} \varphi\right|_{b}^{2} w^{2} \nabla_{N} \varphi\right. \\
& \left.+2 \alpha\left(\nabla_{N} \varphi, \nabla_{N} w\right)_{b} \nabla_{N} w-\alpha\left|\nabla_{N} w\right|_{b}^{2} \nabla_{N} \varphi\right] \\
B_{1}= & 2 s \lambda^{2} \alpha\left|\nabla_{N} \varphi\right|_{b}^{2}-2 s \lambda \alpha \Delta_{N} \varphi\left|\nabla_{N} \varphi\right|_{b}^{2}-s \lambda \alpha\left(\left(\nabla_{N} b\right)\left(\nabla_{N} w, \nabla_{N} w\right), \nabla_{N} \varphi\right)_{b} \\
= & 2 s \lambda^{2} \alpha\left|\nabla_{N} \varphi\right|_{b}^{2}-s \alpha O(\lambda), \\
B_{2}= & 2 s^{3} \lambda^{4} \alpha^{3}\left|\nabla_{N} \varphi\right|_{b}^{4}+2 s^{3} \lambda^{3} \alpha^{3} \operatorname{div}_{N}\left(\left|\nabla_{N} \varphi\right|_{b}^{2} \nabla_{N} \varphi\right)-4 s^{2} \lambda^{2} \alpha^{2}\left|\Delta_{N} \varphi\right|^{2}-4 s^{2} \lambda^{4} \alpha^{2}\left|\nabla_{N} \varphi\right|_{b}^{2} \\
= & 2 s^{3} \lambda^{4} \alpha^{3}\left|\nabla_{N} \varphi\right|_{b}^{4}-s^{3} \alpha^{3} O\left(\lambda^{3}\right)-s^{2} \alpha^{2} O\left(\lambda^{4}\right) .
\end{aligned}\right.
$$


Remark 2.2. There exist several pointwise and weighted estimates for second order partial differential operators in the literature (e.g., $[1,6,10,18,20])$. These estimates are quite useful in control theory and inverse problems for partial differential equations. In [18], Theorem 2.2, one can find an estimate similar to (2.4). The main advantage of our estimate (2.4) consists in that it is more convenient to deal with the Robin boundary condition, as shown in the proof of Theorem 4.1.

Proof of Theorem 2.1. By the definition of $v$ and $w$, we have that

$$
\nabla_{N} v=\nabla_{N}\left(\theta^{-1} w\right)=w \nabla_{N}\left(\theta^{-1}\right)+\theta^{-1} \nabla_{N} w=-s \lambda \theta^{-1} \alpha w \nabla_{N} \varphi+\theta^{-1} \nabla_{N} w .
$$

Hence, by (2.1), it follows that

$$
\begin{aligned}
-\theta \operatorname{div}_{N}\left(\nabla_{N} v\right) & =-\theta \operatorname{div}_{N}\left(-s \lambda \theta^{-1} \alpha w \nabla_{N} \varphi+\theta^{-1} \nabla_{N} w\right) \\
& =-\Delta_{N} w+2 s \lambda \alpha\left(\nabla_{N} \varphi, \nabla_{N} w\right)_{b}+s \lambda^{2} \alpha\left|\nabla_{N} \varphi\right|_{b}^{2} w-s^{2} \lambda^{2} \alpha^{2}\left|\nabla_{N} \varphi\right|_{b}^{2} w+s \lambda \alpha w \Delta_{N} \varphi .
\end{aligned}
$$

Put

$$
\left\{\begin{array}{l}
I_{1}=-\Delta_{N} w-s^{2} \lambda^{2} \alpha^{2}\left|\nabla_{N} \varphi\right|_{b}^{2} w \\
I_{2}=2 s \lambda \alpha\left(\nabla_{N} \varphi, \nabla_{N} w\right)_{b}+2 s \lambda^{2} \alpha\left|\nabla_{N} \varphi\right|_{b}^{2} w, \\
I_{3}=-\theta \Delta_{N} v-s \lambda \alpha w \Delta_{N} \varphi+s \lambda^{2} \alpha\left|\nabla_{N} \varphi\right|_{b}^{2} w .
\end{array}\right.
$$

By (2.7)-(2.8), we see that $I_{1}+I_{2}=I_{3}$. Hence

$$
2 I_{1} I_{2} \leq\left|I_{3}\right|^{2}
$$

We estimate $\left|I_{3}\right|^{2}$ first.

$$
\begin{aligned}
\left|I_{3}\right|^{2} & =\left.\left.\left|-\theta \Delta_{N} v-s \lambda \alpha w \Delta_{N} \varphi+s \lambda^{2} \alpha\right| \nabla_{N} \varphi\right|_{b} ^{2} w\right|^{2} \\
& \leq 2 \theta^{2}\left|\Delta_{N} v\right|^{2}+4 s^{2} \lambda^{2} \alpha^{2}\left|\Delta_{N} \varphi\right|^{2}|w|^{2}+4 s^{2} \lambda^{4} \alpha^{2}\left|\nabla_{N} \varphi\right|_{b}^{4}|w|^{2} .
\end{aligned}
$$

Next, let us estimate $I_{1} I_{2}$. By (2.8), it follows that

$$
\begin{aligned}
I_{1} I_{2}= & 2 s \lambda \alpha\left(-\Delta_{N} w-s^{2} \lambda^{2} \alpha^{2}\left|\nabla_{N} \varphi\right|_{b}^{2} w\right)\left(\left(\nabla_{N} \varphi, \nabla_{N} w\right)_{b}+\lambda\left|\nabla_{N} \varphi\right|_{b}^{2} w\right) \\
= & 2 s \lambda^{2} \alpha\left(-\Delta_{N} w-s^{2} \lambda^{2} \alpha^{2}\left|\nabla_{N} \varphi\right|_{b}^{2} w\right)\left|\nabla_{N} \varphi\right|_{b}^{2} w \\
& -2 s^{3} \lambda^{3} \alpha^{3}\left|\nabla_{N} \varphi\right|_{b}^{2}\left(\nabla_{N} \varphi, \nabla_{N} w\right)_{b} w-2 s \lambda \alpha \Delta_{N} w\left(\nabla_{N} \varphi, \nabla_{N} w\right)_{b} .
\end{aligned}
$$

We need to compute the terms in the right-hand side of (2.11) one by one. By formula (2.1), we find that

$$
\begin{aligned}
2 s \lambda^{2} \alpha\left(-\Delta_{N} w-s^{2} \lambda^{2} \alpha^{2}\left|\nabla_{N} \varphi\right|_{b}^{2} w\right)\left|\nabla_{N} \varphi\right|_{b}^{2} w= & -2 s^{3} \lambda^{4} \alpha^{3}\left|\nabla_{N} \varphi\right|_{b}^{4} w^{2}-\operatorname{div}_{N}\left(2 s \lambda^{2} \alpha\left|\nabla_{N} \varphi\right|_{b}^{2} w \nabla_{N} w\right) \\
& +2 s \lambda^{2}\left(\nabla_{N}\left(\alpha\left|\nabla_{N} \varphi\right|^{2}\right), \nabla_{N} w\right)_{b} w+2 s \lambda^{2} \alpha\left|\nabla_{N} \varphi\right|_{b}^{2}\left|\nabla_{N} w\right|_{b}^{2} .
\end{aligned}
$$

Further,

$$
\begin{aligned}
-2 s^{3} \lambda^{3} \alpha^{3}\left|\nabla_{N} \varphi\right|_{b}^{2}\left(\nabla_{N} \varphi, \nabla_{N} w\right)_{b} w= & -\operatorname{div}_{N}\left(s^{3} \lambda^{3} \alpha^{3}\left|\nabla_{N} \varphi\right|_{b}^{2} w^{2} \nabla_{N} \varphi\right)+3 s^{3} \lambda^{4} \alpha^{3}\left|\nabla_{N} \varphi\right|_{b}^{4} w^{2} \\
& +s^{3} \lambda^{3} \alpha^{3} \operatorname{div}_{N}\left(\left|\nabla_{N} \varphi\right|_{b}^{2} \nabla_{N} \varphi\right) w^{2} .
\end{aligned}
$$

Further,

$$
\begin{aligned}
-2 s \lambda \alpha \Delta_{N} w\left(\nabla_{N} \varphi, \nabla_{N} w\right)_{b}= & -\operatorname{div}_{N}\left(2 s \lambda \alpha\left(\nabla_{N} \varphi, \nabla_{N} w\right)_{b} \nabla_{N} w\right)+2 s \lambda^{2} \alpha\left(\nabla_{N} \varphi, \nabla_{N} w\right)_{b}^{2} \\
& +2 s \lambda \alpha\left(\nabla_{N} w, \nabla_{N}\left(\nabla_{N} w, \nabla_{N} \varphi\right)_{b}\right)_{b} .
\end{aligned}
$$


By formula (2.2),

$$
\begin{aligned}
\left(\nabla_{N} w, \nabla_{N}\left(\nabla_{N} w, \nabla_{N} \varphi\right)_{b}\right)_{b}= & \left(\nabla_{N} w,\left(\left[\nabla_{N}\left(\nabla_{N} w\right)\right], \nabla_{N} \varphi\right)_{b}+\left(\left[\nabla_{N}\left(\nabla_{N} \varphi\right)\right], \nabla_{N} w\right)_{b}\right)_{b} \\
& +\left(\nabla_{N} w,\left(\nabla_{N} b\right)\left(\nabla_{N} w, \nabla_{N} \varphi\right)\right)_{b} .
\end{aligned}
$$

Noting that

$$
2\left(\nabla_{N} w,\left(\left[\nabla_{N}\left(\nabla_{N} w\right)\right], \nabla_{N} \varphi\right)_{b}\right)_{b}=\left(\nabla_{N}\left|\nabla_{N} w\right|_{b}^{2}, \nabla_{N} \varphi\right)_{b}-\left(\left(\nabla_{N} b\right)\left(\nabla_{N} w, \nabla_{N} w\right), \nabla_{N} \varphi\right)_{b}
$$

we arrive at

$$
\begin{aligned}
2 s \lambda \alpha\left(\nabla_{N} w, \nabla_{N}\left(\nabla_{N} w, \nabla_{N} \varphi\right)_{b}\right)_{b}= & s \lambda \alpha\left(\nabla_{N}\left|\nabla_{N} w\right|_{b}^{2}, \nabla_{N} \varphi\right)_{b}-s \lambda \alpha\left(\left(\nabla_{N} b\right)\left(\nabla_{N} w, \nabla_{N} w\right), \nabla_{N} \varphi\right)_{b} \\
& +2 s \lambda \alpha\left(\nabla_{N} w,\left(\left[\nabla_{N}\left(\nabla_{N} \varphi\right)\right], \nabla_{N} w\right)_{b}\right)_{b}+2 s \lambda \alpha\left(\nabla_{N} w,\left(\nabla_{N} b\right)\left(\nabla_{N} w, \nabla_{N} \varphi\right)\right)_{b} \\
= & s \lambda \operatorname{div}_{N}\left(\alpha\left|\nabla_{N} w\right|_{b}^{2} \nabla_{N} \varphi\right)-s \lambda^{2} \alpha\left|\nabla_{N} \varphi\right|_{b}^{2}\left|\nabla_{N} w\right|_{b}^{2}-s \lambda \alpha \Delta_{N} \varphi\left|\nabla_{N} w\right|_{b}^{2} \\
& -s \lambda \alpha\left(\left(\nabla_{N} b\right)\left(\nabla_{N} w, \nabla_{N} w\right), \nabla_{N} \varphi\right)_{b}+2 s \lambda \alpha\left(\nabla_{N} w,\left(\left[\nabla_{N}\left(\nabla_{N} \varphi\right)\right], \nabla_{N} w\right)_{b}\right)_{b} \\
& +2 s \lambda \alpha\left(\nabla_{N} w,\left(\nabla_{N} b\right)\left(\nabla_{N} w, \nabla_{N} \varphi\right)\right)_{b} .
\end{aligned}
$$

Therefore it holds

$$
\begin{aligned}
-2 s \lambda \alpha \Delta_{N} w\left(\nabla_{N} \varphi, \nabla_{N} w\right)_{b}= & -\operatorname{div}_{N}\left(2 s \lambda \alpha\left(\nabla_{N} \varphi, \nabla_{N} w\right)_{b} \nabla_{N} w\right)+2 s \lambda^{2} \alpha\left(\nabla_{N} \varphi, \nabla_{N} w\right)_{b}^{2} \\
& +\operatorname{div}_{N}\left(s \lambda \alpha\left|\nabla_{N} w\right|_{b}^{2} \nabla_{N} \varphi\right)-s \lambda \alpha\left(\left(\nabla_{N} b\right)\left(\nabla_{N} w, \nabla_{N} w\right), \nabla_{N} \varphi\right)_{b} \\
& -s \lambda^{2} \alpha\left|\nabla_{N} \varphi\right|_{b}^{2}\left|\nabla_{N} w\right|_{b}^{2}-s \lambda \alpha \Delta_{N} \varphi\left|\nabla_{N} w\right|_{b}^{2}+2 s \lambda \alpha\left(\nabla_{N} w,\left(\left[\nabla_{N}\left(\nabla_{N} \varphi\right)\right], \nabla_{N} w\right)_{b}\right)_{b} \\
& +2 s \lambda \alpha\left(\nabla_{N} w,\left(\nabla_{N} b\right)\left(\nabla_{N} w, \nabla_{N} \varphi\right)\right)_{b} .
\end{aligned}
$$

Finally, by (2.9)-(2.14), we obtain (2.4).

\section{A nONNEGATIVE FUnCtion With AN ARBITRARY GIVEN CRITICAL POINT LOCATION IN THE MANIFOLD}

In this section, we prove the existence of a nonnegative function with an arbitrary given critical point location in manifold $M$. This result is a modification of the corresponding result in [2] for flat spaces. In the sequel, this construction will play a key role in the choice of the weight function in our global Carleman estimate.

Our result is stated as follows:

Theorem 3.1. There exists a function $\psi \in C^{2}(M)$ such that $\psi>0$ in $M, \psi=0$ on $\Gamma$ and

$$
\left|\nabla_{M} \psi\right|_{g}^{2}>0, \quad \forall x \in \overline{M \backslash \omega_{0}}
$$

where $\omega_{0}$ is an arbitrary fixed nonempty open subset of $M$ such that $\overline{\omega_{0}} \subset \omega$.

Proof of Theorem 3.1. We borrow some idea from [2]. Choose a function $p \in C^{2}(M)$ such that

$$
p>0 \text { in } M, p=0 \text { and }\left|\nabla_{M} p\right|_{g}>0 \text { on } \Gamma \text {. }
$$

By the density of Morse functions in $C^{2}(M)$ (see [17], Chap. 1), there exists a sequence of Morse functions $\left\{p^{k}(x)\right\}_{k=1}^{\infty}$ such that

$$
p^{k} \rightarrow p \text { in } C^{2}(M), \text { as } k \rightarrow \infty \text {. }
$$


Denote by $M_{1}=\left\{x \in M \mid \nabla_{M} p(x)=0\right\}$ the set of critical points of function $p$. Since $\left|\nabla_{M} p\right|_{g}>0$ on $\partial M$, there exist a positive number $\xi_{1}>0$ and an open set $M_{2} \subset M$ such that

$$
\left|\nabla_{M} p\right|_{g}>\xi_{1}>0 \text { in } M_{2}, M_{1} \cap \overline{M_{2}}=\emptyset, \Gamma \subset \overline{M_{2}} .
$$

Let $f \in C^{\infty}(M)$ such that

$$
f=1 \text { on } \Gamma, f=0 \text { in } M \backslash \overline{M_{2}} .
$$

Put $q^{k}(x)=p^{k}(x)+f(x)\left[p(x)-p^{k}(x)\right]$. By the definition of $q^{k}$, we know

$$
q^{k}=0 \text { on } \Gamma, \nabla_{M} q^{k}=\nabla_{M} p^{k} \text { in } M \backslash \overline{M_{2}}
$$

and

$$
\nabla_{M} q^{k}(x)=\nabla_{M} p^{k}(x)+f(x)\left[\nabla_{M} p(x)-\nabla_{M} p^{k}(x)\right]+\nabla_{M} f(x)\left[p(x)-p^{k}(x)\right] .
$$

By (3.3), we know that there exists a $\bar{k} \in \mathbb{N}$ such that for any integer $k>\bar{k}$, we have

$$
f(x)\left[\nabla_{M} p(x)-\nabla_{M} p^{k}(x)\right]+\nabla_{M} f(x)\left[p(x)-p^{k}(x)\right]<\frac{\xi_{1}}{2} .
$$

From (3.4), (3.7) and (3.8), for any integer $k_{1}>\bar{k}$, it follows that

$$
\left|\nabla_{M} q^{k_{1}}\right|_{g}>0 \text { in } \overline{M_{2}} .
$$

Letting $q(x)=q^{k_{1}}(x)$, we know that $q$ is a Morse function satisfying $\left|\nabla_{M} q\right|_{g}>0$ in $\overline{M_{2}}$.

Denote by $C P_{1}$ the set of critical points of function q, i.e., $C P_{1}=\left\{x \in M \mid \nabla_{M} q(x)=0\right\}$. Hence $C P_{1}$ is a finite set. Assume $C P_{1}=\left\{x_{1}, x_{2}, \ldots, x_{m}\right\}$. Consider a sequence of functions $\left\{\rho^{i}\right\}_{i=1}^{m} \subset C^{\infty}([0,1] ; M)$ such that

$$
\left\{\begin{array}{l}
\rho^{i}(t) \in M, \forall t \in[0,1], \rho^{i}\left(t_{1}\right) \neq \rho^{i}\left(t_{2}\right), \forall t_{1}, t_{2} \in[0,1], t_{1} \neq t_{2}, i=1, \ldots, m, \\
\rho^{i}(1)=x_{i}, \rho^{i}(0) \in \omega_{1}, i=1, \ldots, m, \\
\rho^{i}\left(t_{1}\right) \neq \rho^{j}\left(t_{2}\right), \forall i \neq j, \forall t_{1}, t_{2} \in[0,1],
\end{array}\right.
$$

where $\omega_{1}$ is a nonempty open set such that $\overline{\omega_{1}} \subset \omega_{0}$. By (3.10), there exists a sequence of $C^{2}$-vector fields $\left\{\eta^{i}\right\}_{i=1}^{m}$ on $M$ and a sequence of $C^{\infty}$-functions $\left\{\gamma^{i}\right\}_{i=1}^{m}$ on $M$ such that

$$
\begin{aligned}
\frac{\mathrm{d} \rho^{i}(t)}{d t} & =\eta^{i}\left(\rho^{i}(t)\right), \quad \text { in }[0,1], i=1, \ldots, m, \\
\operatorname{supp} \gamma^{i} \subset M, i & =1, \ldots, m, \\
\operatorname{supp} \gamma^{i} \cap \operatorname{supp} \gamma^{j} & =\emptyset, \forall i \neq j, \\
\gamma^{i}\left(\rho^{i}(t)\right) & =1, \forall t \in[0,1], i=1, \ldots, m .
\end{aligned}
$$

Let $V^{i}(x)=\gamma^{i}(x) \eta^{i}(x)$. Consider the system of the ordinary differential equations on manifold $M$ as follows:

$$
\left\{\begin{array}{l}
\frac{\mathrm{d} x}{\mathrm{~d} t}=V^{i}(x), \\
x(0)=x_{0} .
\end{array}\right.
$$

Denote by $S_{t}^{i}: M \rightarrow M(i=1, \ldots, m)$ the operator such that $S_{t}^{i}\left(x_{0}\right)=x(t)$, where $x(t)$ is the solution of equation (3.15). Hence $S_{t}^{i}(i=1, \ldots, m)$ are diffeomorphisms on $M$.

By (3.10), (3.11) and (3.14), we have

$$
S_{1}^{i}\left(\rho^{i}(0)\right)=x_{i}, i=1, \ldots, m .
$$


Put $S(x)=S_{1}^{1} \circ S_{1}^{2} \circ \ldots \circ S_{1}^{m}$ and $\psi(x)=q(S(x))$. By (3.12), there exists a domain $M_{3} \subset M$ such that $\Gamma \subset \overline{M_{3}}$ and

$$
S_{1}^{i}(x)=x, \forall x \in M_{3}, i=1, \ldots, m .
$$

Therefore $\psi(x)=q(x), \forall x \in M_{3}$. Hence $\psi(x)=0, \forall x \in \partial M$. Denote by $C P_{2}$ the critical points of $\psi$. Since the mapping $S$ is a diffeomorphism, we have

$$
C P_{2}=\left\{x \in M \mid S(x) \in C P_{1}\right\} .
$$

By (3.13), we have

$$
S\left(\rho^{i}(0)\right)=x_{i}, i=1, \ldots, m .
$$

It follows from (3.18) and (3.19) that $C P_{2} \subset \omega_{0}$, which completes the proof.

\section{INTERPOLATION INEQUALITY I}

This section is devoted to showing an interpolation result for solutions to the following elliptic equation:

$$
\left\{\begin{array}{l}
u_{t t}+\Delta_{M} u=0 \quad \text { in } Q \\
\frac{\partial_{M} u}{\partial \nu}+l(x) u=0 \text { on } \Sigma .
\end{array}\right.
$$

Our result reads:

Theorem 4.1. Let $0<\gamma<\frac{T}{2}$ and $2 \gamma<T^{\prime}<T^{\prime \prime}<T-\gamma$. Then there exists a constant $\mu \in(0,1)$ such that any solution $u \in H^{2}(Q)$ of (4.1) satisfies

$$
|u|_{L^{2}\left(M \times\left(T^{\prime}, T^{\prime \prime}\right)\right)} \leq C|u|_{L^{2}(\omega \times(\gamma, T-\gamma))}^{\mu}|u|_{H^{1}(Q)}^{1-\mu} .
$$

This sort of interpolation estimate has already appeared in the framework of boundary control and stabilization for hyperbolic equations (e.g. [8]) and also for inverse problems (e.g. [18]).

Proof of Theorem 4.1. We borrow some ideas from [18]. The key is to use Theorem 2.1. The proof is divided into five steps.

Step 1. Firstly, we will explain the construction of the weight function $\theta$ appeared in Theorem 2.1. By (3.1), we have

$$
h \triangleq \frac{1}{|\psi|_{L^{\infty}(M)}} \min _{x \in \overline{M \backslash \omega_{0}}}|\nabla \psi(x)|_{g}>0 .
$$

Without loss of generality, let us assume that $T^{\prime} \leq T-T^{\prime \prime}$. Let

$$
a=\frac{T}{2}-2 \gamma, \quad a_{0}=\frac{T-T^{\prime}-2 \gamma}{2}, \quad a_{1}=\frac{T}{2}-\gamma .
$$

It is easy to check that

$$
\frac{T}{2}-T^{\prime}<a_{0}<a<a_{1}<\frac{T}{2}
$$

We choose

$$
\varphi(x, t)=\left(c_{1}-c_{2}\right) \frac{\psi(x)}{|\psi|_{L^{\infty}(M)}}+a^{2}-\left(t-\frac{T}{2}\right)^{2}+\kappa
$$

and

$$
\widetilde{\varphi}(x, t)=-\left(c_{1}-c_{2}\right) \frac{\psi(x)}{|\psi|_{L^{\infty}(M)}}+a^{2}-\left(t-\frac{T}{2}\right)^{2}+\kappa,
$$


where $c_{1}=a^{2}-\left(\frac{T}{2}-T^{\prime}\right)^{2}, c_{2}=a^{2}-\frac{1}{2}\left(\frac{T}{2}-T^{\prime}\right)^{2}-a_{0}^{2}$ and $\kappa$ is chosen to be large enough to make $\widetilde{\varphi}>0$. It is easy to check that $c_{1}>c_{2}$.

These give the functions $\alpha(x, t)=\mathrm{e}^{\lambda \varphi(x, t)}, \widetilde{\alpha}(x, t)=\mathrm{e}^{\lambda \widetilde{\varphi}(x, t)}, \theta=\mathrm{e}^{s \alpha}$ and $\widetilde{\theta}=\mathrm{e}^{s \widetilde{\alpha}}$. It is obvious that $0<\widetilde{\varphi} \leq \varphi, 1<\widetilde{\alpha} \leq \alpha$ and $1<\widetilde{\theta} \leq \theta$.

By the definition of $\alpha$, it is easy to check that

$$
\begin{cases}\alpha(\cdot, t) \geq \mathrm{e}^{c_{1} \lambda+\lambda \kappa}, & \left|t-\frac{T}{2}\right| \leq \frac{T}{2}-T^{\prime} \\ \alpha(\cdot, t) \leq \mathrm{e}^{\left(c_{1}-c_{2}\right) \lambda+\lambda \kappa}, & \left|t-\frac{T}{2}\right| \geq a\end{cases}
$$

Noting that equation (4.1) has only partial boundary condition. We need to reduce it into an equation with full boundary condition. For this, let us choose a cut-off function $\phi(t) \in C_{0}^{\infty}\left(\frac{T}{2}-a_{1}, \frac{T}{2}+a_{1}\right)=C_{0}^{\infty}(\gamma, T-\gamma)$ such that

$$
\begin{cases}0 \leq \phi(t) \leq 1, & t \in(\gamma, T-\gamma) \\ \phi(t)=1, & \left|t-\frac{T}{2}\right| \leq \frac{T}{2}-a .\end{cases}
$$

Let $u_{1}=\phi u$, noticing that $\phi$ is independent of $x$, it follows by equation (4.1) that

$$
\begin{cases}\left(u_{1}\right)_{t t}+\Delta_{M} u_{1}=\phi_{t t} u+2 \phi_{t} u_{t} & \text { in } Q, \\ \frac{\partial_{M} u_{1}}{\partial \nu}+l(x) u_{1}=0 & \text { on } \Sigma, \\ u_{1}=0 & \text { on }(M \times\{0\}) \cup(M \times\{T\}) .\end{cases}
$$

By (4.8), we know that there is a $Q_{0} \subset Q$ such that

$$
\left\{\begin{array}{l}
\operatorname{supp}\left(u_{1}\right) \subset Q_{0} \\
\partial Q_{0} \text { is } C^{2}
\end{array}\right.
$$

Put $\Sigma_{0}=\partial Q_{0} \cap \Sigma$.

Step 2. We now apply Theorem 2.1 to equation (4.9) with $n=d+1, N=Q_{0}, b=1 \otimes g, v$ being replaced by $u_{1}, \varphi$ is as $(4.5)$ and $w=\theta u_{1}$.

Integrating equality (2.4) on $Q_{0}$, we obtain that

$$
\begin{aligned}
& \int_{Q_{0}} 2 \theta^{2}\left|\left(u_{1}\right)_{t t}+\Delta_{M} u_{1}\right|^{2} d_{g} x \mathrm{~d} t+\int_{Q_{0}} D d_{g} x \mathrm{~d} t \\
\geq & \int_{Q_{0}} B_{1}\left|\nabla_{N} w\right|_{b}^{2} d_{g} x \mathrm{~d} t+\int_{Q_{0}} B_{2} w^{2} d_{g} x \mathrm{~d} t+4 s \lambda^{2} \int_{Q_{0}}\left(\nabla_{N}\left(\alpha\left|\nabla_{N} \varphi\right|_{b}^{2}\right), \nabla_{N} w\right)_{b} w d_{g} x \mathrm{~d} t \\
\quad+ & 4 s \lambda^{2} \int_{Q_{0}} \alpha\left(\nabla_{N} w, \nabla_{N} \varphi\right)_{b}^{2} d_{g} x \mathrm{~d} t+4 s \lambda \int_{Q_{0}} \alpha\left(\nabla_{N} w,\left[\nabla_{N}\left(\nabla_{N} \varphi\right)\right] \nabla_{N} w\right)_{b} d_{g} x \mathrm{~d} t \\
& +4 s \lambda \int_{Q_{0}} \alpha\left(\nabla_{N} w,\left(\nabla_{N} b\right)\left(\nabla_{N} w, \nabla_{N} \varphi\right)\right)_{b} d_{g} x \mathrm{~d} t-2 s \lambda \int_{Q_{0}} \alpha\left(\left(\nabla_{N} b\right)\left(\nabla_{N} w, \nabla_{N} w\right), \nabla_{N} \varphi\right)_{b} d_{g} x \mathrm{~d} t .
\end{aligned}
$$

Let us estimate the right-hand side of (4.11). By Cauchy-Schwarz inequality and noting that $\varphi \in C^{2}\left(Q_{0}\right)$, we have the following estimates:

$$
\begin{aligned}
4 s \lambda^{2}\left|\left(\nabla_{N}\left(\alpha\left|\nabla_{N} \varphi\right|_{b}^{2}\right), \nabla_{N} w\right)_{b} w\right| & \leq C\left(s^{2} \lambda^{4} \alpha w^{2}+\lambda^{2}\left|\nabla_{M} w\right|_{g}^{2}+\lambda^{2}\left|w_{t}\right|^{2}\right), \\
4 s \lambda\left|\alpha\left(\nabla_{N} w,\left[\nabla_{N}\left(\nabla_{N} \varphi\right)\right] \nabla_{N} w\right)_{b}\right| & \leq C s \lambda \alpha\left(\left|\nabla_{M} w\right|_{g}^{2}+\left|w_{t}\right|^{2}\right), \\
4 s \lambda\left|\alpha\left(\nabla_{N} w,\left(\nabla_{N} b\right)\left(\nabla_{N} w, \nabla_{N} \varphi\right)\right)_{b}\right| & \leq C s \lambda \alpha\left(\left|\nabla_{M} w\right|_{g}^{2}+\left|w_{t}\right|^{2}\right), \\
s \lambda\left|\alpha\left(\left(\nabla_{N} b\right)\left(\nabla_{N} w, \nabla_{N} w\right), \nabla_{N} \varphi\right)_{b}\right| & \leq C s \lambda \alpha\left(\left|\nabla_{M} w\right|_{g}^{2}+\left|w_{t}\right|^{2}\right) .
\end{aligned}
$$


By the definition of $B_{1}$, we have that

$$
B_{1}\left|\nabla_{N} w\right|_{b}^{2}=\left[2 s \lambda^{2} \alpha\left|\nabla_{N} \varphi\right|_{b}^{2}-s \alpha O(\lambda)\right]\left(\left|\nabla_{M} w\right|_{g}^{2}+\left|w_{t}\right|^{2}\right) .
$$

By the definition of $B_{2}$, we have that

$$
B_{2} w^{2}=\left[2 s^{3} \lambda^{4} \alpha^{3}\left|\nabla_{N} \varphi\right|_{b}^{4}-s^{3} \alpha^{3} O\left(\lambda^{3}\right)-s^{2} \alpha^{2} O(\lambda)^{4}\right] w^{2} .
$$

Recalling (4.5) for the definition of $\varphi$ and (4.3) for the positive constant $h$, we conclude that there is a constant $\lambda_{0}>1$ such that for any $\lambda \geq \lambda_{0}$, one can find a constant $s_{0}>1$ so that for any $s \geq s_{0}$, the following estimates hold uniformly for $(x, t) \in \overline{M \times(2-a, 2+a) \backslash \omega_{0} \times\left(2-a_{0}, 2+a_{0}\right)}$ :

$$
\left\{\begin{array}{l}
B_{1}\left|\nabla_{N} w\right|_{b}^{2}-C\left(s \lambda \alpha+\lambda^{2} \alpha\right)\left(\left|\nabla_{M} w\right|_{g}^{2}+\left|w_{t}\right|^{2}\right) \geq\left(c_{1}-c_{2}\right)^{2} h^{2} s \lambda^{2} \alpha\left(\left|\nabla_{M} w\right|_{g}^{2}+\left|w_{t}\right|^{2}\right), \\
B_{2} w^{2}-C s^{2} \lambda^{4} \alpha w^{2} \geq\left(c_{1}-c_{2}\right)^{4} h^{4} s^{3} \lambda^{4} \alpha^{3}|w|^{2} .
\end{array}\right.
$$

From (4.11) and (4.18), we conclude that

$$
\begin{array}{r}
s \lambda^{2} \int_{Q_{0}} \alpha\left(\left|\nabla_{M} w\right|_{g}^{2}+\left|w_{t}\right|^{2}\right) d_{g} x \mathrm{~d} t+s^{3} \lambda^{4} \int_{Q_{0}} \alpha^{3}|w|^{2} d_{g} x \mathrm{~d} t \leq C\left\{\int_{Q_{0}} \theta^{2}\left|\left(u_{1}\right)_{t t}+\Delta_{M} u_{1}\right|^{2} d_{g} x \mathrm{~d} t+\int_{Q_{0}} D d_{g} x \mathrm{~d} t\right. \\
\left.+s \lambda^{2} \int_{0}^{T} \int_{\omega_{0}} \alpha\left(\left|\nabla_{M} w\right|_{g}^{2}+\left|w_{t}\right|^{2}\right) d_{g} x \mathrm{~d} t+s^{3} \lambda^{4} \int_{0}^{T} \int_{\omega_{0}} \alpha^{3}|w|^{2} d_{g} x \mathrm{~d} t\right\}
\end{array}
$$

Step 3. We now get rid of the boundary term $\int_{Q_{0}} D d_{g} x \mathrm{~d} t$ in (4.19).

Using the divergence theorem and the boundary condition of equation (4.9), the first term in $\int_{Q_{0}} D d_{g} x \mathrm{~d} t$ reads

$$
4 s \lambda^{2} \int_{\Sigma_{0}} \alpha\left|\nabla_{N} \varphi\right|_{b}^{2} w \frac{\partial_{M} w}{\partial \nu} d \Gamma_{g} \mathrm{~d} t=4 s \lambda^{2} \int_{\Sigma_{0}} \alpha\left(\left|\nabla_{M} \varphi\right|_{g}^{2}+\left|\varphi_{t}\right|^{2}\right)\left(s \lambda \alpha \frac{\partial_{M} \varphi}{\partial \nu} w^{2}-l w^{2}\right) d_{g} \Gamma \mathrm{d} t .
$$

The second one is

$$
2 s^{3} \lambda^{3} \int_{\Sigma_{0}} \alpha^{3}\left(\left|\nabla_{M} \varphi\right|_{g}^{2}+\left|\varphi_{t}\right|^{2}\right) \frac{\partial_{M} \varphi}{\partial \nu} w^{2} d_{g} \Gamma \mathrm{d} t
$$

The third one is

$$
4 s \lambda \int_{\Sigma_{0}} \alpha\left(\nabla_{N} \varphi, \nabla_{N} w\right)_{b} \frac{\partial_{M} w}{\partial \nu} d_{g} \Gamma \mathrm{d} t=4 s \lambda \int_{\Sigma_{0}} \alpha\left[\left(\nabla_{M} \varphi, \nabla_{M} w\right)_{g}+\varphi_{t} w_{t}\right]\left(s \lambda \alpha w \frac{\partial_{M} \varphi}{\partial \nu}+\theta \frac{\partial_{M} u_{1}}{\partial \nu}\right) d_{g} \Gamma \mathrm{d} t
$$

By the boundary condition of $u_{1}$, we have that $\frac{\partial_{M} u_{1}}{\partial \nu}=-l u_{1}$. Especially, noting that $\left.\psi\right|_{\Gamma}=0$, we have that $\left.\nabla_{M} \varphi\right|_{\Gamma}=\left.\frac{\partial_{M} \varphi}{\partial \nu} \nu\right|_{\Gamma}$. Hence from (4.22), we get that

$$
\begin{aligned}
& 4 s \lambda \int_{\Sigma_{0}} \alpha\left(\nabla_{N} \varphi, \nabla_{N} w\right)_{b} \frac{\partial_{M} w}{\partial \nu} d_{g} \Gamma \mathrm{d} t \\
= & \int_{\Sigma_{0}}\left(4 s^{3} \lambda^{3} \alpha^{3}\left|\nabla_{M} \varphi\right|_{g}^{2} \frac{\partial_{M} \varphi}{\partial \nu} w^{2}-8 s^{2} \lambda^{2} \alpha^{2}\left|\nabla_{M} \varphi\right|_{g}^{2} l w^{2}+4 s \lambda \alpha \frac{\partial_{M} \varphi}{\partial \nu} l^{2} w^{2}\right) d_{g} \Gamma \mathrm{d} t \\
& +4 s^{2} \lambda^{2} \int_{\Sigma_{0}} \alpha^{2} \varphi_{t} w_{t} w \frac{\partial_{M} \varphi}{\partial \nu} d_{g} \Gamma \mathrm{d} t-4 s \lambda \int_{\Sigma_{0}} \alpha \varphi_{t} l w_{t} w d_{g} \Gamma \mathrm{d} t .
\end{aligned}
$$


By integration by parts, we get that

$$
\begin{aligned}
-4 s \lambda \int_{\Sigma_{0}} \alpha \varphi_{t} w_{t} \theta l u_{1} d_{g} \Gamma \mathrm{d} t & =-4 s \lambda \int_{\Sigma_{0}} \theta \alpha \varphi_{t}\left(s \lambda \alpha \varphi_{t} \theta u_{1}+\theta\left(u_{1}\right)_{t}\right) l u_{1} d_{g} \Gamma \mathrm{d} t \\
& =2 s \lambda \int_{\Sigma_{0}}\left\{\lambda \alpha \varphi_{t}^{2} l w^{2}+\alpha \varphi_{t t} l w^{2}\right\} d_{g} \Gamma \mathrm{d} t .
\end{aligned}
$$

Therefore, we obtain that

$$
\begin{aligned}
& 4 s \lambda \int_{\Sigma_{0}} \alpha\left(\nabla_{N} \varphi, \nabla_{N} w\right)_{b} \frac{\partial_{M} w}{\partial \nu} d_{g} \Gamma \mathrm{d} t \\
= & \int_{\Sigma_{0}}\left(4 s^{3} \lambda^{3} \alpha^{3}\left|\nabla_{M} \varphi\right|_{g}^{2} \frac{\partial_{M} \varphi}{\partial \nu} w^{2}-8 s^{2} \lambda^{2} \alpha^{2}\left|\nabla_{M} \varphi\right|_{g}^{2} l w^{2}+4 s \lambda \alpha \frac{\partial_{M} \varphi}{\partial \nu} l^{2} w^{2}\right) d_{g} \Gamma \mathrm{d} t \\
& +4 s^{2} \lambda^{2} \int_{\Sigma_{0}} \alpha^{2} \varphi_{t} w_{t} w \frac{\partial_{M} \varphi}{\partial \nu} d_{g} \Gamma \mathrm{d} t+\int_{\Sigma_{0}}\left\{2 s^{2} \lambda^{2} \alpha^{2} \varphi_{t}^{2} l w^{2}-2 s \lambda^{2} \alpha \varphi_{t}^{2} l w^{2}-2 s \lambda \alpha \varphi_{t t} l w^{2}\right\} d_{g} \Gamma \mathrm{d} t .
\end{aligned}
$$

The fourth one is

$$
\begin{aligned}
& -2 \int_{\Sigma_{0}} s \lambda \alpha\left|\nabla_{N} w\right|_{b}^{2} \frac{\partial_{M} \varphi}{\partial \nu} d_{g} \Gamma \mathrm{d} t \\
= & -2 \int_{\Sigma_{0}} s \lambda \alpha\left(\left|\nabla_{M} w\right|_{g}^{2}+w_{t}^{2}\right) \frac{\partial_{M} \varphi}{\partial \nu} d_{g} \Gamma \mathrm{d} t \\
= & -2 \int_{\Sigma_{0}}\left[s^{3} \lambda^{3} \alpha^{3}\left|\nabla_{M} \varphi\right|_{g}^{2} \frac{\partial_{M} \varphi}{\partial \nu} w^{2}-2 s^{2} \lambda^{2} \alpha^{2}\left|\nabla_{M} \varphi\right|_{g}^{2} l w^{2}+s \lambda \alpha \theta^{2}\left|\nabla_{M} u_{1}\right|_{g}^{2} \frac{\partial_{M} \varphi}{\partial \nu}\right] d_{g} \Gamma \mathrm{d} t \\
& -2 \int_{\Sigma_{0}} s \lambda \alpha w_{t}^{2} \frac{\partial_{M} \varphi}{\partial \nu} d_{g} \Gamma \mathrm{d} t .
\end{aligned}
$$

Therefore we have

$$
\begin{aligned}
\int_{Q_{0}} D d_{g} x \mathrm{~d} t= & \int_{\Sigma_{0}}\left[4 s^{2} \lambda^{3} \alpha^{2}\left(\left|\nabla_{M} \varphi\right|_{g}^{2}+\left|\varphi_{t}\right|^{2}\right) w^{2}+4 s^{3} \lambda^{3} \alpha^{3}\left|\nabla_{M} \varphi\right|_{g}^{2} w^{2}+2 s^{3} \lambda^{3} \alpha^{3}\left|\varphi_{t}\right|^{2} w^{2}\right. \\
& \left.+4 s \lambda \alpha l^{2} w^{2}+4 s^{2} \lambda^{2} \alpha^{2} \varphi_{t} w w_{t}-2 s \lambda \alpha \theta^{2}\left|\nabla_{M} u_{1}\right|_{g}^{2}-2 s \lambda \alpha l w_{t}^{2}\right] \frac{\partial_{M} \varphi}{\partial \nu} d_{g} \Gamma \mathrm{d} t \\
& -\int_{\Sigma_{0}}\left[4 s \lambda^{2} \alpha\left|\nabla_{M} \varphi\right|_{g}^{2} l(1+s \alpha)-2 s \lambda^{2} \alpha l\left|\varphi_{t}\right|^{2}+2 s \lambda \alpha l \varphi_{t t}\right] w^{2} d_{g} \Gamma \mathrm{d} t .
\end{aligned}
$$

Since $\left.\left|\nabla_{M} \varphi\right|_{g}\right|_{\Gamma}>0$, we know that there exists an $s_{1}>0$ such that for all $s>s_{1}$, we have that

$$
\int_{\Sigma_{0}}\left[4 s \lambda^{2} \alpha\left|\nabla_{M} \varphi\right|_{g}^{2} l(1+s \alpha)-2 s \lambda^{2} \alpha l\left|\varphi_{t}\right|^{2}+2 s \lambda \alpha l \varphi_{t t}\right] w^{2} d_{g} \Gamma \mathrm{d} t \geq 0 .
$$

Hence the right-hand side of (4.27) could be divided into two parts. The second integral is negative and has the property we expect. We need only to deal with the first integral in the right hand side of (4.27). We now 
choose another weight function $\widetilde{\theta}$. By (4.5), (4.6) and noting that $\psi$ vanishes on $\Gamma$, we have the following equalities:

$$
\left.\varphi\right|_{\Sigma}=\left.\widetilde{\varphi}\right|_{\Sigma},\left.\quad \frac{\partial_{M} \varphi}{\partial \nu}\right|_{\Sigma}=-\left.\frac{\partial_{M} \widetilde{\varphi}}{\partial \nu}\right|_{\Sigma},\left.\alpha\right|_{\Sigma}=\left.\widetilde{\alpha}\right|_{\Sigma},\left.\quad w\right|_{\Sigma}=\left.\widetilde{w}\right|_{\Sigma},\left.\quad w_{t}\right|_{\Sigma}=\left.\widetilde{w}_{t}\right|_{\Sigma}
$$

Similar to (4.19) and (4.27), we deduce the following inequality:

$$
\begin{aligned}
s \lambda^{2} \int_{Q_{0}} \widetilde{\alpha}\left(\left|\nabla_{M} \widetilde{w}\right|_{g}^{2}+\left|\widetilde{w}_{t}\right|^{2}\right) d_{g} x \mathrm{~d} t+s^{3} \lambda^{4} \int_{Q_{0}} \widetilde{\alpha}^{3}|\widetilde{w}|^{2} d_{g} x \mathrm{~d} t \leq C\left\{\int_{Q_{0}} \widetilde{\theta}^{2}\left|\left(u_{1}\right)_{t t}+\Delta_{M} u_{1}\right|^{2} d_{g} x \mathrm{~d} t\right. \\
\left.+\int_{Q_{0}} \widetilde{D} d_{g} x \mathrm{~d} t+s \lambda^{2} \int_{0}^{T} \int_{\omega_{0}} \widetilde{\alpha}\left(\left|\nabla_{M} \widetilde{w}\right|_{g}^{2}+\left|\widetilde{w}_{t}\right|^{2}\right) d_{g} x \mathrm{~d} t+s^{3} \lambda^{4} \int_{0}^{T} \int_{\omega_{0}} \widetilde{\alpha}^{3}|\widetilde{w}|^{2} d_{g} x \mathrm{~d} t\right\}
\end{aligned}
$$

where $\widetilde{w}=\widetilde{\theta} u_{1}$ and

$$
\begin{aligned}
\int_{Q_{0}} \widetilde{D} d_{g} x \mathrm{~d} t= & -\int_{\Sigma_{0}}\left[4 s^{2} \lambda^{3} \alpha^{2}\left(\left|\nabla_{M} \varphi\right|_{g}^{2}+\left|\varphi_{t}\right|^{2}\right) w^{2}+6 s^{3} \lambda^{3} \alpha^{3}\left|\nabla_{M} \varphi\right|_{g}^{2} w^{2}+4 s^{3} \lambda^{3} \alpha^{3}\left|\varphi_{t}\right|^{2} w^{2}\right. \\
& \left.+4 s \lambda \alpha l_{1}^{2} w^{2}+4 s^{2} \lambda^{2} \alpha^{2} \varphi_{t} w w_{t}-2 s \lambda \alpha \theta^{2}\left|\nabla_{M} u_{1}\right|_{g}^{2}-2 s \lambda \alpha l w_{t}^{2}\right] \frac{\partial_{M} \varphi}{\partial \nu} d_{g} \Gamma \mathrm{d} t \\
& -\int_{\Sigma_{0}}\left[4 s \lambda^{2} \alpha\left|\nabla_{M} \varphi\right|_{g}^{2} l(1+s \alpha)-2 s \lambda^{2} \alpha l\left|\varphi_{t}\right|^{2}-2 s \lambda \alpha l \varphi_{t t}\right] w^{2} d_{g} \Gamma \mathrm{d} t .
\end{aligned}
$$

From (4.27) and (4.31), we know that for $\forall s>s_{1}$, we have that

$$
\int_{Q_{0}}(D+\widetilde{D}) d_{g} x \mathrm{~d} t=-\int_{\Sigma_{0}}\left[8 s \lambda^{2} \alpha\left|\nabla_{M} \varphi\right|_{g}^{2} l(1+s \alpha)-4 s \lambda^{2} \alpha l\left|\varphi_{t}\right|^{2}-4 s \lambda \alpha l \varphi_{t t}\right] w^{2} d_{g} \Gamma \mathrm{d} t \leq 0 .
$$

By (4.19), (4.30) and noting (4.32), we arrive at

$$
\begin{aligned}
& s \lambda^{2} \int_{Q_{0}}\left[\alpha\left(\left|\nabla_{M} w\right|_{g}^{2}+\left|w_{t}\right|^{2}\right)+\widetilde{\alpha}\left(\left|\nabla_{M} \widetilde{w}\right|_{g}^{2}+\left|\widetilde{w}_{t}\right|^{2}\right)\right] d_{g} x \mathrm{~d} t,+s^{3} \lambda^{4} \int_{Q_{0}}\left(\alpha^{3}|w|^{2}+\widetilde{\alpha}^{3}|\widetilde{w}|^{2}\right) d_{g} x \mathrm{~d} t \\
\leq & C\left\{\int_{Q_{0}}\left(\theta^{2}\left|\left(u_{1}\right)_{t t}+\Delta_{M} u_{1}\right|^{2}+\widetilde{\theta}^{2}\left|\left(u_{1}\right)_{t t}+\Delta_{M} u_{1}\right|^{2}\right) d_{g} x \mathrm{~d} t+s^{3} \lambda^{4} \int_{0}^{T} \int_{\omega_{0}}\left(\alpha^{3}|w|^{2}+v \widetilde{\alpha}^{3}|\widetilde{w}|^{2}\right) d_{g} x \mathrm{~d} t\right. \\
& \left.+s \lambda^{2} \int_{0}^{T} \int_{\omega_{0}}\left[\alpha\left(\left|\nabla_{M} w\right|_{g}^{2}+\left|w_{t}\right|^{2}\right)+\widetilde{\alpha}\left(\left|\nabla_{M} \widetilde{w}\right|_{g}^{2}+\left|\widetilde{w}_{t}\right|^{2}\right)\right] d_{g} x \mathrm{~d} t\right\} .
\end{aligned}
$$

Step 4. We now return both $w$ and $\widetilde{w}$ in (4.33) to $u_{1}$. Recalling that $w=\theta u_{1}$ and $\widetilde{w}=\widetilde{\theta} u_{1}$, we obtain that

$$
\begin{aligned}
\frac{1}{C} \theta^{2}\left(\left|\nabla_{M} u_{1}\right|_{g}^{2}+\left|\left(u_{1}\right)_{t}\right|^{2}+s^{2} \lambda^{2} \alpha^{2}\left|u_{1}\right|^{2}\right) & \leq\left|\nabla_{M} w\right|_{g}^{2}+\left|w_{t}\right|^{2}+s^{2} \lambda^{2} \alpha^{2} w^{2} \\
& \leq C \theta^{2}\left(\left|\nabla_{M} u_{1}\right|_{g}^{2}+\left|\left(u_{1}\right)_{t}\right|^{2}+s^{2} \lambda^{2} \alpha^{2}\left|u_{1}\right|^{2}\right)
\end{aligned}
$$


and

$$
\begin{aligned}
\frac{1}{C} \widetilde{\theta}^{2}\left(\left|\nabla_{M} u_{1}\right|_{g}^{2}+\left|\left(u_{1}\right)_{t}\right|^{2}+s^{2} \lambda^{2} \widetilde{\alpha}^{2}\left|u_{1}\right|^{2}\right) & \leq\left|\nabla_{M} \widetilde{w}\right|_{g}^{2}+\left|\widetilde{w}_{t}\right|^{2}+s^{2} \lambda^{2} \widetilde{\alpha}^{2} \widetilde{w}^{2} \\
& \leq C \widetilde{\theta}^{2}\left(\left|\nabla_{M} u_{1}\right|_{g}^{2}+\left|\left(u_{1}\right)_{t}\right|^{2}+s^{2} \lambda^{2} \alpha^{2}\left|u_{1}\right|^{2}\right) .
\end{aligned}
$$

By the definition of $\alpha, \widetilde{\alpha}, \theta$ and $\widetilde{\theta}$, we know $\alpha \geq \widetilde{\alpha}>1$ and $\theta \geq \widetilde{\theta}>1$. Hence, by (4.33)-(4.35), we end up with the following inequality:

$$
\begin{aligned}
s^{3} \lambda^{4} \int_{Q_{0}} \alpha^{3} \theta^{2}\left|u_{1}\right|^{2} d_{g} x \mathrm{~d} t \leq & C\left\{\int_{Q_{0}} \theta^{2}\left|\Delta_{M} u_{1}+\left(u_{1}\right)_{t t}\right|^{2} d_{g} x \mathrm{~d} t+s^{3} \lambda^{4} \int_{0}^{T} \int_{\omega_{0}} \alpha^{3} \theta^{2}\left|u_{1}\right|^{2} d_{g} x \mathrm{~d} t\right. \\
& \left.+s \lambda^{2} \int_{0}^{T} \int_{\omega_{0}} \alpha \theta^{2}\left(\left|\nabla_{M} u_{1}\right|_{g}^{2}+\left|\left(u_{1}\right)_{t}\right|^{2}\right) d_{g} x \mathrm{~d} t\right\} .
\end{aligned}
$$

Recalling that $u_{1}$ is the solution of equation (4.9), we know

$$
\left|\Delta_{M} u_{1}+\left(u_{1}\right)_{t t}\right|^{2} \leq\left|\phi_{t t} u+2 \phi_{t} u_{t}\right|^{2} \text {. }
$$

Choose a cut-off function $g \in C_{0}^{\infty}(\omega)$ with $g=1$ in $\omega_{0}$ and $0 \leq g \leq 1$ in $\omega$. Multiplying equation (4.9) by $g \theta^{2} \alpha u_{1}$ and integrating it in $Q_{0}$, using integration by parts, we get

$$
\int_{0}^{T} \int_{\omega_{0}} \alpha \theta^{2}\left(\left|\nabla_{M} u_{1}\right|_{g}^{2}+\left|\left(u_{1}\right)_{t}\right|^{2}\right) d_{g} x \mathrm{~d} t \leq C\left[s^{2} \lambda^{2} \int_{0}^{T} \int_{\omega} \theta^{2} \alpha^{2}\left|u_{1}\right|^{2} d_{g} x \mathrm{~d} t+\int_{Q_{0}} \theta^{2}\left|\phi_{t t} u+2 \phi_{t} u_{t}\right|^{2} d_{g} x \mathrm{~d} t\right] .
$$

From (4.36)-(4.38), we obtain

$$
s^{3} \lambda^{4} \int_{Q_{0}} \alpha^{3} \theta^{2}\left|u_{1}\right|^{2} d_{g} x \mathrm{~d} t \leq C\left\{s \lambda^{2} \int_{Q_{0}} \theta^{2}\left|\phi_{t t} u+2 \phi_{t} u_{t}\right|^{2} d_{g} x \mathrm{~d} t+s^{3} \lambda^{4} \int_{0}^{T} \int_{\omega} \alpha^{3} \theta^{2}\left|u_{1}\right|^{2} d_{g} x \mathrm{~d} t\right\} .
$$

Step 5. Finally, we shall drop the weight functions in the integrands of (4.39) to get the desired result. Noting that $\alpha$ satisfies (4.7) and $\varphi$ satisfies (4.8), we have the following inequalities:

$$
\begin{aligned}
\int_{Q_{0}} s^{3} \lambda^{4} \alpha^{3} \theta^{2} u_{1}^{2} d_{g} x \mathrm{~d} t \geq & s^{3} \lambda^{4} \mathrm{e}^{3\left(c_{1} \lambda+\lambda \kappa\right)} \mathrm{e}^{2 s\left(\mathrm{e}^{c_{1} \lambda+\lambda \kappa}\right)} \int_{T^{\prime}}^{T^{\prime \prime}} \int_{M}|u|^{2} d_{g} x \mathrm{~d} t \\
\int_{Q_{0}} \theta^{2}\left|\phi_{t t} u+2 \phi_{t} u_{t}\right|^{2} d_{g} x \mathrm{~d} t= & \int_{\frac{T}{2}-a_{1}}^{\frac{T}{2}+a_{1}} \int_{M} \theta^{2}\left|\phi_{t t} u+2 \phi_{t} u_{t}\right|^{2} d_{g} x \mathrm{~d} t \\
\leq & C \mathrm{e}^{2 s \mathrm{e}^{\left(c_{1}-c_{2}\right) \lambda+\lambda \kappa}}\left\{\int_{\frac{T}{2}-a_{1}}^{\frac{T}{2}-a} \int_{M}\left(|u|^{2}+\left|u_{t}\right|^{2}\right) d_{g} x \mathrm{~d} t\right. \\
& \left.+\int_{\frac{T}{2}+a}^{\frac{T}{2}+a_{1}} \int_{M}\left(|u|^{2}+\left|u_{t}\right|^{2}\right) d_{g} x \mathrm{~d} t\right\} \\
\leq & C \mathrm{e}^{2 s \mathrm{e}^{\left(c_{1}-c_{2}\right) \lambda+\lambda \kappa}}|u|_{H^{1}(Q)}^{2},
\end{aligned}
$$

and

$$
\begin{aligned}
\int_{0}^{T} \int_{\omega} s^{3} \lambda^{4} \alpha^{3} \theta^{2}\left|u_{1}\right|^{2} d_{g} x \mathrm{~d} t & =\int_{\frac{T}{2}-a_{1}}^{\frac{T}{2}+a_{1}} \int_{\omega} s^{3} \lambda^{4} \alpha^{3} \theta^{2}\left|u_{1}\right|^{2} d_{g} x \mathrm{~d} t \\
& \leq s^{3} \lambda^{4} \mathrm{e}^{3 \lambda\left(\frac{T^{2}}{4}+c_{1}-c_{2}+\kappa\right)} \mathrm{e}^{2 s \mathrm{e}^{\lambda\left(\frac{T^{2}}{4}+c_{1}-c_{2}+\kappa\right)}} \int_{\gamma}^{T-\gamma} \int_{\omega}|u|^{2} d_{g} x \mathrm{~d} t .
\end{aligned}
$$


From (4.39)-(4.42), we obtain

$$
\begin{aligned}
s^{3} \lambda^{4} \mathrm{e}^{3\left(c_{1} \lambda+\lambda \kappa\right)} \mathrm{e}^{2 s\left(\mathrm{e}^{c_{1} \lambda+\lambda \kappa}\right)} \int_{T^{\prime}}^{T^{\prime \prime}} \int_{M}|u|^{2} d_{g} x \mathrm{~d} t \leq & s^{3} \lambda^{4} \mathrm{e}^{3 \lambda\left(\frac{T^{2}}{4}+c_{1}-c_{2}+\kappa\right)} \mathrm{e}^{2 s \mathrm{e}^{\lambda\left(\frac{T^{2}}{4}+c_{1}-c_{2}+\kappa\right)}} \\
& \times \int_{\gamma}^{T-\gamma} \int_{\omega}|u|^{2} d_{g} x \mathrm{~d} t+C \mathrm{e}^{2 s \mathrm{e}^{\left(c_{1}-c_{2}\right) \lambda+\lambda \kappa}}|u|_{H^{1}(Q)}^{2} .
\end{aligned}
$$

Recalling that $c_{1}>c_{2}>0$, hence we know that $\mathrm{e}^{2 s\left(\mathrm{e}^{c_{1} \lambda+\lambda \kappa}\right)}>\mathrm{e}^{2 s\left(\mathrm{e}^{\left(c_{1}-c_{2}\right) \lambda+\lambda \kappa}\right)}$. Let $\lambda=\lambda_{0}$,

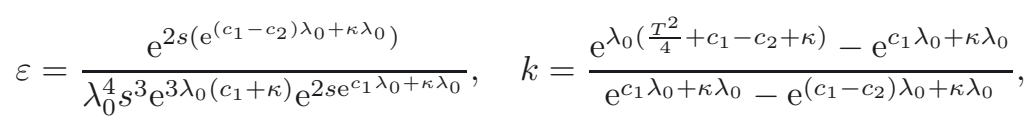

and

$$
\varepsilon_{0}=\frac{\mathrm{e}^{2 s_{0}\left(\mathrm{e}^{\left(c_{1}-c_{2}\right) \lambda_{0}+\kappa \lambda_{0}}\right)}}{\lambda_{0}^{4} s_{0}^{3} \mathrm{e}^{3 \lambda_{0}\left(c_{1}+\kappa\right)} \mathrm{e}^{2 s_{0} \mathrm{e}^{c_{1} \lambda_{0}+\kappa \lambda_{0}}}} .
$$

From (4.43), we know that for any $\varepsilon \in\left(0, \varepsilon_{0}\right]$, it holds

$$
|u|_{L^{2}\left(M \times\left(T^{\prime}, T^{\prime \prime}\right)\right)}^{2} \leq \varepsilon^{-k}|u|_{L^{2}(\omega \times(\gamma, T-\gamma))}^{2}+C \varepsilon|u|_{H^{1}(Q)}^{2},
$$

which in turn implies that the above inequality holds for any $\varepsilon>0$.

Let $\mu=\frac{1}{1+k}, \varepsilon=\left(\frac{|u|_{L^{2}(\omega \times(\gamma, T-\gamma))}}{|u|_{H^{1}(Q)}}\right)^{2 \mu}$, by inequality (4.44), we get

$$
|u|_{L^{2}\left(M \times\left(T^{\prime}, T^{\prime \prime}\right)\right)} \leq C|u|_{L^{2}(\omega \times(\gamma, T-\gamma))}^{\mu}|u|_{H^{1}(M \times(0, T))}^{1-\mu} .
$$

\section{INTERPOLATION INEQUALITY II}

This section is devoted to showing another interpolation result for solutions to equation (4.1). Our result is stated as follows:

Theorem 5.1. Let $0<\gamma<\frac{T}{2}$. Then there exists a constant $\delta \in(0,1)$ such that any solution $u \in H^{2}(Q)$ of (4.1) satisfies

$$
|u|_{H^{1}(\omega \times(\gamma, T-\gamma))} \leq C\left(|u(0)|_{L^{2}(\omega)}+\left|u_{t}(0)\right|_{L^{2}(\omega)}+\left|\nabla_{M} u(0)\right|_{L^{2}(\omega)}\right)^{\delta}|u|_{H^{1}(Q)}^{1-\delta} .
$$

Proof of Theorem 5.1. We divide the proof into three steps.

Step 1. Let $\omega_{2} \subset \subset \omega$. Denote by dist $\left((x, t), \omega_{2} \times\{0\}\right)$ the distance from $(x, t)$ to $\omega_{1} \times\{0\}$. Put $N(\tau)=\{(x, t) \in$ $\left.Q \mid \operatorname{dist}\left((x, t), \omega_{2} \times\{0\}\right)<\tau\right\}$. Let $0<\tau_{1}<\tau_{2}<\tau_{3}$ such that $N\left(\tau_{3}\right) \subset Q$ and $N\left(\tau_{3}\right) \cap(M \times\{0\}) \subset \omega \times\{0\}$.

Let $h$ be an $C^{2}$-function such that

$$
\left\{\begin{array}{l}
3<h<4 \quad \text { if }(x, t) \in N\left(\tau_{1}\right) \\
0<h<1 \quad \text { if }(x, t) \in N\left(\tau_{3}\right) \backslash N\left(\tau_{2}\right), \\
\left|\nabla_{M} h\right|>0 \text { for } \operatorname{all}(x, t) \in N\left(\tau_{3}\right) .
\end{array}\right.
$$

The construction of $h$ is very easy. For example, we can choose a smooth function $h_{1}: \mathbb{R} \rightarrow \mathbb{R}$ such that

$$
\begin{cases}h_{1}^{\prime}<0 \text { and } 3<h_{1}(s)<4 & \text { if } 0<s<\tau_{1}^{2}, \\ 0<h_{1}(s)<1 & \text { if } \tau_{2}^{2}<s<\tau_{3}^{2} .\end{cases}
$$

Then $h(x, t)=h_{1}\left(\operatorname{dist}^{2}\left((x, t), \omega_{2} \times\{0\}\right)\right)$ is the desired function. 
In what follows, we shall use Theorem 2.1 (with $\varphi$ replaced by $h$ ) to prove Theorem 5.1. For simplicity of the notations, we still use $\theta$ to denote the weight function if there is no confusion.

Denote by $d_{b} \partial N\left(\tau_{3}\right)$ the volume element of $\partial N\left(\tau_{3}\right)$ in its Riemannian metric and by $\bar{\nu}=\bar{\nu}(x)$ the unit outward normal vector of $N\left(\tau_{3}\right)$ at $x \in \partial N\left(\tau_{3}\right)$ with its Riemannian metric.

For $\varepsilon$ small enough, define

$$
N_{\varepsilon}\left(\partial\left(N\left(\tau_{3}\right)\right) \backslash\left(\omega_{2} \times\{0\}\right)\right) \triangleq\left\{x: x \in N\left(\tau_{3}\right), \operatorname{dist}\left((x, t), \partial\left(N\left(\tau_{3}\right)\right) \backslash\left(\omega_{2} \times\{0\}\right)\right)<\varepsilon\right\} .
$$

Choose a function $\chi \in C^{\infty}\left(N\left(\tau_{3}\right)\right)$ such that $0 \leq \chi \leq 1$ and that

$$
\chi= \begin{cases}1 & \text { if }(x, t) \in N\left(\tau_{2}\right), \\ 0 & \text { if }(x, t) \in N\left(\tau_{3}\right) \cap N_{\varepsilon}\left(\partial\left(N\left(\tau_{3}\right)\right) \backslash\left(\omega_{2} \times\{0\}\right)\right) .\end{cases}
$$

Put $\bar{u}=\chi u$ where $u$ is the solution of equation (4.1). Then, $\bar{u}$ satisfies the following equation:

$$
\begin{cases}\bar{u}_{t t}+\Delta_{M} \bar{u}=\chi_{t t} u+2 \chi_{t} u_{t}+u \Delta_{M} \chi+2\left(\nabla_{M} u, \nabla_{M} \chi\right)_{g} & \text { in } N\left(\tau_{3}\right), \\ \left|\nabla_{M} \bar{u}\right|_{g}=\bar{u}=0 & \text { on } \partial N\left(\tau_{3}\right) \backslash(\omega \times\{0\}) .\end{cases}
$$

Apply Theorem 2.1 to equation (5.2) with $b=1 \otimes g, v$ replaced by $\bar{u}$ and $w=\theta \bar{u}$.

Proceeding as in (4.12)-(4.18), similar to (4.19), and noting that $h$ has no critical point in $N\left(\tau_{3}\right)$, we obtain that

$$
\begin{aligned}
& s \lambda^{2} \int_{N\left(\tau_{3}\right)} \alpha\left(\left|\nabla_{M} w\right|_{g}^{2}+\left|w_{t}\right|^{2}\right) d_{g} x \mathrm{~d} t+s^{3} \lambda^{4} \int_{N\left(\tau_{3}\right)} \alpha^{3}|w|^{2} d_{g} x \mathrm{~d} t \\
\leq & C\left\{\int_{N\left(\tau_{3}\right)} \theta^{2}\left|\bar{u}_{t t}+\Delta_{M} \bar{u}\right|^{2} d_{g} x \mathrm{~d} t+\int_{N\left(\tau_{3}\right)} D_{1} d_{g} x \mathrm{~d} t\right\},
\end{aligned}
$$

where

$D_{1}=2 s \lambda \operatorname{div}_{N}\left[2 \lambda \alpha\left|\nabla_{N} h\right|_{b}^{2} w \nabla_{N} w+s^{2} \lambda^{2} \alpha^{3}\left|\nabla_{N} h\right|_{b}^{2} w^{2} \nabla_{N} h+2 \alpha\left(\nabla_{N} h, \nabla_{N} w\right)_{b} \nabla_{N} w-\alpha\left|\nabla_{N} w\right|_{b}^{2} \nabla_{N} h\right]$.

By the divergence theorem, $\int_{N\left(\tau_{3}\right)} D_{1} d_{g} x \mathrm{~d} t$ is the boundary term.

For the first term therein, we have

$$
\int_{\partial N\left(\tau_{3}\right)} 2 s \lambda^{2} \alpha\left|\nabla_{N} h\right|_{b}^{2} w \frac{\partial w}{\partial \bar{\nu}} d_{b} \partial N\left(\tau_{3}\right) \leq C \int_{\partial N\left(\tau_{3}\right)}\left\{s \lambda \alpha\left(\left|\nabla_{M} w\right|_{g}^{2}+w_{t}^{2}\right)+s \lambda^{3} \alpha w^{2}\right\} d_{b} \partial N\left(\tau_{3}\right) .
$$

Due to the definition of $w$, we know

$$
\left\{\begin{array}{l}
\left.w\right|_{\partial N\left(\tau_{3}\right) \backslash(\omega \times\{0\})}=\left.\bar{u}\right|_{\partial N\left(\tau_{3}\right) \backslash(\omega \times\{0\})}=0, \\
\left.\nabla_{N} w\right|_{\partial N\left(\tau_{3}\right) \backslash(\omega \times\{0\})}=\left.\nabla_{N} \bar{u}\right|_{\partial N\left(\tau_{3}\right) \backslash(\omega \times\{0\})}=0 .
\end{array}\right.
$$

Hence we know that

$$
\int_{\partial N\left(\tau_{3}\right)} 2 s \lambda^{2} \alpha\left|\nabla_{N} h\right|_{b}^{2} w \frac{\partial w}{\partial \bar{\nu}} d_{b} \partial N\left(\tau_{3}\right) \leq C \int_{\omega \times\{0\}}\left\{s \lambda \alpha\left(\left|\nabla_{M} w\right|_{g}^{2}+w_{t}^{2}\right)+s \lambda^{3} \alpha w^{2}\right\} d_{g} x .
$$

By the same argument, we obtain the estimates for the remainder terms. Therefore, it follows that

$$
\int_{Q} D_{1} d_{g} x \mathrm{~d} t \leq C \int_{\omega \times\{0\}}\left[s \lambda \alpha\left(\left|\nabla_{M} w\right|_{g}^{2}+w_{t}^{2}\right)+s^{3} \lambda^{3} \alpha^{3} w^{2}\right] d_{g} x .
$$


Combining (5.3) and (5.5), we obtain that

$$
\begin{aligned}
s \lambda^{2} \int_{N\left(\tau_{3}\right)} \alpha\left(\left|\nabla_{M} w\right|_{g}^{2}+\left|w_{t}\right|^{2}\right) d_{g} x \mathrm{~d} t+s^{3} \lambda^{4} \int_{N\left(\tau_{3}\right)} & \alpha^{3}|w|^{2} d_{g} x \mathrm{~d} t \leq C\left\{\int_{N\left(\tau_{3}\right)} \theta^{2}\left|\bar{u}_{t t}+\Delta_{M} \bar{u}\right|^{2} d_{g} x \mathrm{~d} t\right. \\
& \left.+\int_{\omega \times\{0\}}\left[s \lambda \alpha\left(\left|\nabla_{M} w\right|_{g}^{2}+\left|w_{t}\right|^{2}\right)+s^{3} \lambda^{3} \alpha^{3}|w|^{2}\right] d_{g} x\right\} .
\end{aligned}
$$

Step 2. We now return the $w$ in (5.6) to $\bar{u}$. Recalling $w=\theta \bar{u}$, it is clear that

$$
\begin{aligned}
\frac{1}{C} \theta^{2}\left(\left|\nabla_{M} \bar{u}\right|_{g}^{2}+\left|\bar{u}_{t}\right|^{2}+s^{2} \lambda^{2} \alpha^{2}|\bar{u}|^{2}\right) & \leq\left|\nabla_{M} w\right|_{g}^{2}+\left|w_{t}\right|^{2}+s^{2} \lambda^{2} \alpha^{2} w^{2} \\
& \leq C \theta^{2}\left(\left|\nabla_{M} \bar{u}\right|_{g}^{2}+\left|\bar{u}_{t}\right|^{2}+s^{2} \lambda^{2} \alpha^{2}|\bar{u}|^{2}\right) .
\end{aligned}
$$

From (5.6)-(5.7), and noting the first equation in (5.2), we obtain that

$$
\begin{aligned}
& s \lambda^{2} \int_{N\left(\tau_{3}\right)} \alpha \theta^{2}\left(\left|\nabla_{M} \bar{u}\right|_{g}^{2}+\left|\bar{u}_{t}\right|^{2}\right) d_{g} x \mathrm{~d} t+s^{3} \lambda^{4} \int_{N\left(\tau_{3}\right)} \alpha^{3} \theta^{2}|\bar{u}|^{2} d_{g} x \mathrm{~d} t \\
\leq & C\left\{\int_{N\left(\tau_{3}\right)} \theta^{2}\left|\chi_{t t} u+2 \chi_{t} u_{t}+u \Delta_{M} \chi+2\left(\nabla_{M} u, \nabla_{M} \chi\right)_{g}\right|^{2} d_{g} x \mathrm{~d} t+\int_{\omega \times\{0\}}\left[s \lambda \alpha\left(\left|\nabla_{M} \bar{u}\right|_{g}^{2}+\bar{u}_{t}^{2}\right)+s^{3} \lambda^{3} \alpha^{3} \bar{u}^{2}\right] d_{g} x\right\} .
\end{aligned}
$$

By the definition of $\bar{u}$, we know that $\bar{u}=u$ in $N\left(\tau_{1}\right)$. By the definition of $h$, we know that

$$
\begin{cases}\alpha \geq \mathrm{e}^{3 \lambda} \text { and } \theta \geq \mathrm{e}^{s \mathrm{e}^{3 \lambda}} & \text { if }(x, t) \in N\left(\tau_{1}\right), \\ \alpha \leq \mathrm{e}^{\lambda} \text { and } \theta \leq \mathrm{e}^{s \mathrm{e}^{\lambda}} & \text { if }(x, t) \in N\left(\tau_{3}\right) \backslash N\left(\tau_{2}\right) .\end{cases}
$$

By the definition of $\chi$ we know that

$$
\chi_{t}=0 \text { and } \nabla_{M} \chi=0 \text { if }(x, t) \in N\left(\tau_{2}\right) .
$$

Therefore we have the following inequalities:

$$
\begin{gathered}
\int_{N\left(\tau_{3}\right)} s \lambda^{2} \alpha \theta^{2}\left(\left|\nabla_{M} \bar{u}\right|_{g}^{2}+\left|\bar{u}_{t}\right|^{2}\right) d_{g} x \mathrm{~d} t \geq s \lambda^{2} \mathrm{e}^{3 \lambda} \mathrm{e}^{2 s \mathrm{e}^{3 \lambda}} \int_{N\left(\tau_{1}\right)}\left(\left|\nabla_{M} \bar{u}\right|_{g}^{2}+\left|\bar{u}_{t}\right|^{2}\right) d_{g} x \mathrm{~d} t \\
\int_{N\left(\tau_{3}\right)} s^{3} \lambda^{4} \alpha^{3} \theta^{2} \bar{u}^{2} d_{g} x \mathrm{~d} t \geq s^{3} \lambda^{4} \mathrm{e}^{9 \lambda} \mathrm{e}^{2 s \mathrm{e}^{3 \lambda}} \int_{N\left(\tau_{1}\right)}|u|^{2} d_{g} x \mathrm{~d} t \\
\int_{N\left(\tau_{3}\right)} \theta^{2} \mid \chi_{t t} u+2 \chi_{t} u_{t}+u \Delta_{M} \chi+ \\
\left.2\left(\nabla_{M} u, \nabla_{M} \chi\right)_{g}\right|^{2} d_{g} x \mathrm{~d} t \leq C \mathrm{e}^{2 s \mathrm{e}^{\lambda}} \int_{N\left(\tau_{3}\right)}\left(|u|^{2}+\left|\nabla_{M} u\right|_{g}^{2}+\left|u_{t}\right|^{2}\right) d_{g} x \mathrm{~d} t \\
\int_{\omega \times\{0\}} s^{3} \lambda^{3} \alpha^{3} \theta^{2}|\bar{u}|^{2} d_{g} x \leq s^{3} \lambda^{3} \mathrm{e}^{12 \lambda} \mathrm{e}^{2 s \mathrm{e}^{4 \lambda}} \int_{\omega \times\{0\}}|u|^{2} d_{g} x
\end{gathered}
$$

and

$$
\int_{\omega \times\{0\}} s \lambda \alpha \theta^{2}\left(\left|\nabla_{M} \bar{u}\right|_{g}^{2}+\bar{u}_{t}^{2}\right) d_{g} x \leq s \lambda \mathrm{e}^{4 \lambda} \mathrm{e}^{2 s \mathrm{e}^{4 \lambda}} \int_{\omega \times\{0\}}\left(\left|\nabla_{M} u\right|_{g}^{2}+u_{t}^{2}\right) d_{g} x
$$


From (5.8) to (5.13), we know that

$$
\begin{aligned}
& s \lambda^{2} \mathrm{e}^{3 \lambda} \mathrm{e}^{2 s \mathrm{e}^{3 \lambda}} \int_{N\left(\tau_{1}\right)}\left(\left|\nabla_{M} \bar{u}\right|_{g}^{2}+\left|\bar{u}_{t}\right|^{2}\right) d_{g} x \mathrm{~d} t+s^{3} \lambda^{4} \mathrm{e}^{9 \lambda} \mathrm{e}^{2 s \mathrm{e}^{3 \lambda}} \int_{N\left(\tau_{1}\right)} s^{3} \lambda^{4}|u|^{2} d_{g} x \mathrm{~d} t \\
\leq & C\left\{\mathrm{e}^{2 s \mathrm{e}^{\lambda}} \int_{N\left(\tau_{3}\right)}\left(|u|^{2}+\left|\nabla_{M} u\right|_{g}^{2}+\left|u_{t}\right|^{2}\right) d_{g} x \mathrm{~d} t+s^{3} \lambda^{3} \mathrm{e}^{12 \lambda} \mathrm{e}^{2 s \mathrm{e}^{4 \lambda}} \int_{\omega \times\{0\}}|u|^{2} d_{g} x\right. \\
& \left.+s \lambda \mathrm{e}^{4 \lambda} \mathrm{e}^{2 s \mathrm{e}^{4 \lambda}} \int_{\omega \times\{0\}}\left(\left|\nabla_{M} u\right|_{g}^{2}+u_{t}^{2}\right) d_{g} x\right\} .
\end{aligned}
$$

Similar to (4.44), by (5.14), we obtain that there exist a $\beta>0$ and an $\epsilon_{0}>0$ such that for any $\epsilon \in\left(0, \epsilon_{0}\right]$, we have

$$
|u|_{H^{1}\left(N\left(\tau_{1}\right)\right)}^{2} \leq \epsilon^{-\beta}\left(\left|u_{t}(0)\right|_{L^{2}(\omega)}^{2}+\left|\nabla_{M} u(0)\right|_{L^{2}(\omega)}^{2}+|u(0)|_{L^{2}(\omega)}^{2}\right)+C \epsilon|u|_{H^{1}(Q)}^{2},
$$

which in turn implies that the above inequality holds for any $\epsilon>0$.

Noting that $\tau_{1}>0$, hence there is an open ball $B \subset N\left(\tau_{1}\right)$. Then we know that

$$
|u|_{H^{1}(B)}^{2} \leq \epsilon^{-\beta}\left(\left|u_{t}(0)\right|_{L^{2}(\omega)}^{2}+\left|\nabla_{M} u(0)\right|_{L^{2}(\omega)}^{2}+|u(0)|_{L^{2}(\omega)}^{2}\right)+C \epsilon|u|_{H^{1}(Q)}^{2} .
$$

Put $\delta^{\prime}=\frac{1}{1+\beta}$ and let $\epsilon=\left(\frac{\left|u_{t}(0)\right|_{L^{2}(\omega)}^{2}+\left|\nabla_{M} u(0)\right|_{L^{2}(\omega)}^{2}+|u(0)|_{L^{2}(\omega)}^{2}}{|u|_{H^{1}(Q)}^{2}}\right)^{\delta^{\prime}}$ in (5.16), we get

$$
\begin{aligned}
|u|_{H^{1}(B)} & \leq C\left(\left|u_{t}(0)\right|_{L^{2}(\omega)}^{2}+\left|\nabla_{M} u(0)\right|_{L^{2}(\omega)}^{2}+|u(0)|_{L^{2}(\omega)}^{2}\right)^{\frac{\delta^{\prime}}{2}}|u|_{H^{1}(Q)}^{1-\delta^{\prime}} \\
& \leq C\left(\left|u_{t}(0)\right|_{L^{2}(\omega)}+\left|\nabla_{M} u(0)\right|_{L^{2}(\omega)}+|u(0)|_{L^{2}(\omega)}\right)^{\delta^{\prime}}|u|_{H^{1}(Q)}^{1-\delta^{\prime}} .
\end{aligned}
$$

Step 3. To complete the proof, it suffices to show that the following proposition: For any given open set $L \subset \subset Q$, there exists a constant $0<\delta^{\prime \prime}<1$ such that

$$
|u|_{H^{1}(L)} \leq C|u|_{H^{1}(B)}^{\delta^{\prime \prime}}|u|_{H^{1}(Q)}^{1-\delta^{\prime \prime}} .
$$

Firstly, we admit this claim and continue our proof. After that, we prove this proposition.

By inequality (5.17) and (5.18), we deduce that for any given subset $L \subset \subset Q$, we have

$$
|u|_{H^{1}(L)} \leq C\left(\left|u_{t}(0)\right|_{L^{2}(\omega)}+\left|\nabla_{M} u(0)\right|_{L^{2}(\omega)}+|u(0)|_{L^{2}(\omega)}\right)^{\delta}|u|_{H^{1}(Q)}^{1-\delta}
$$

where $\delta=\delta^{\prime} \delta^{\prime \prime}$. Now we choose $L=\omega \times(\gamma, T-\gamma)$ to get Theorem 5.1.

Now we prove the above proposition. Let $B_{1}, B_{2}$ and $B_{3}$ be three open balls in $Q$ such that $B_{1} \subset \subset B_{2} \subset \subset$ $B_{3} \subset \subset Q$. Choose a cut-off function $\eta \in C_{0}^{\infty}(Q)$ such that $\eta=1$ in $B_{3}$ and $0<\eta<1$. Let $y=\eta u$. Then, $y$ solves

$$
\begin{cases}y_{t t}+\Delta_{M} y=\eta_{t t} u+2 \eta_{t} u_{t}+u \Delta_{M} \eta+2\left(\nabla_{M} u, \nabla_{M} \eta\right)_{g} & \text { in } Q, \\ \left|\nabla_{M} y\right|=\bar{y}=0 & \text { on } \partial Q .\end{cases}
$$

Denote by $P$ the center of $B_{1}$. Let $r(x, t)=\operatorname{dist}^{2}((x, t), P)$. Replace the above $\varphi($ in $\theta)$ by $r$. By the same argument as the proof of Theorem 4.1, we conclude that there exists a constant $0<\widetilde{\delta}<1$ such that

$$
|u|_{H^{1}\left(B_{2}\right)} \leq C|u|_{H^{1}\left(B_{1}\right)}^{\widetilde{\widetilde{f}}}|u|_{H^{1}(Q)}^{1-\widetilde{\delta}} .
$$


For any ball $B^{\prime} \subset \subset Q$, we can find a finite number $m \in \mathbb{N}$ and two sequences of balls $\left\{B^{i}\right\}_{i=1}^{m}$ and $\left\{\widetilde{B}^{i}\right\}_{i=1}^{m}$ such that

$$
\left\{\begin{array}{l}
B^{\prime} \subset \subset B^{1}, \\
\widetilde{B}^{i} \subset \subset B^{i} \cap B^{i+1} \quad \text { for } i=1, \ldots, m-1, \\
\widetilde{B}^{n} \subset \subset B^{n} \\
\widetilde{B}^{n}=B .
\end{array}\right.
$$

By inequality (5.21), we know that there exists a sequences $\left\{\widetilde{\delta}_{i}\right\}_{i=1}^{m}$ satisfying $0<\widetilde{\delta}_{i}<1$ for $i=1, \ldots, m$, such that

$$
\begin{aligned}
|u|_{H^{1}\left(B^{\prime}\right)} & \leq|u|_{H^{1}\left(B^{1}\right)} \leq C|u|_{H^{1}\left(\widetilde{B}^{1}\right)}^{\widetilde{\delta}_{1}}|u|_{H^{1}(Q)}^{1-\widetilde{\delta}_{1}} \leq C|u|_{H^{1}\left(B^{2}\right)}^{\widetilde{\delta}_{1}}|u|_{H^{1}(Q)}^{1-\widetilde{\delta}_{1}} \\
& \leq C|u|_{H^{1}\left(\widetilde{B}^{2}\right)}^{\widetilde{\delta}_{\delta^{\prime}} \widetilde{\delta}_{2}}|u|_{H^{1}(Q)}^{1-\widetilde{\delta}_{1} \widetilde{\delta}_{2}} \leq \ldots \leq C|u|_{H^{1}\left(\widetilde{B}^{n}\right)}^{\widetilde{\delta}_{1} \widetilde{\delta}_{2} \widetilde{\delta}_{m}}|u|_{H^{1}(Q)}^{1-\widetilde{\delta}_{1} \widetilde{\delta}_{2} \ldots \widetilde{\delta}_{m}}
\end{aligned}
$$

Put $\widetilde{\widetilde{\delta}}=\widetilde{\delta}_{1} \widetilde{\delta}_{2} \ldots \widetilde{\delta}_{m}$, then we know that

$$
|u|_{H^{1}\left(B^{\prime}\right)} \leq C|u|_{H^{1}(B)}^{\widetilde{\widetilde{\delta}}}|u|_{H^{1}(Q)}^{1-\tilde{\widetilde{\delta}}}
$$

For any given $L \subset \subset Q$, we can find finite balls contained in the internal of $Q$ to cover it. Hence from inequality (5.24), we know that there exist a constant $0<\delta^{\prime \prime}<1$ such that (5.18) holds.

$$
|u|_{H^{1}(L)} \leq C|u|_{H^{1}(B)}^{\delta^{\prime \prime}}|u|_{H^{1}(Q)}^{1-\delta^{\prime \prime}} .
$$

This completes the proof of Theorem 5.1.

\section{Proof of Theorem 1.2}

We are now in a position to prove Theorem 1.2.

Proof of Theorem 1.2. For simplicity, choose $T=4, T^{\prime}=1$ and $T^{\prime \prime}=3$ in inequalities (4.2) and (5.1). From Theorem 4.1, we get

$$
|u|_{L^{2}(M \times(1,3))} \leq C|u|_{L^{2}(\omega \times(\gamma, 4-\gamma))}^{\mu}|u|_{H^{1}(Q)}^{1-\mu} .
$$

By Theorem 5.1, we obtain

$$
|u|_{L^{2}(M \times(1,3))} \leq C\left(\left|u_{t}(0)\right|_{L^{2}(\omega)}^{2}+\left|\nabla_{M} u(0)\right|_{L^{2}(\omega)}^{2}+|u(0)|_{L^{2}(\omega)}^{2}\right)^{\mu \delta}|u|_{H^{1}(Q)}^{1-\mu \delta},
$$

where $u \in H^{2}(Q)$ is any solution of equation (4.1).

For any $\left\{a_{i}\right\}_{\lambda_{i} \leq r}$ with $a_{i} \in \mathbb{C}$, set

$$
y(x, t)=\sum_{\lambda_{j} \leq r} \frac{\operatorname{sh}\left(t \sqrt{\lambda_{j}}\right)}{\sqrt{\lambda_{j}}} a_{j} e_{j}
$$

with $\frac{\operatorname{sh}(t b)}{b}=t$ if $b=0$. Then, both the real part and the imaginary part of $y$ are solutions of (4.1) and $\operatorname{Re} y=\operatorname{Im} y=0$ on $M \times\{0\}$. Therefore $\operatorname{Re} y$ and $\operatorname{Im} y$ satisfy inequality (6.2). For the left term of (6.2), we have

$$
\begin{aligned}
|\operatorname{Re} y|_{L^{2}(M \times(1,3))}^{2} & =\int_{1}^{3} \int_{M}\left|\sum_{\lambda_{j} \leq r} \frac{\operatorname{sh}\left(t \sqrt{\lambda_{j}}\right)}{\sqrt{\lambda_{j}}}\left(\operatorname{Re} a_{j}\right) e_{j}\right|^{2} \mathrm{~d} x \mathrm{~d} t=\sum_{\lambda_{j} \leq r}\left|\operatorname{Re} a_{j}\right|^{2} \int_{1}^{3}\left|\frac{\operatorname{sh}\left(t \sqrt{\lambda_{j}}\right)}{\sqrt{\lambda_{j}}}\right|^{2} \mathrm{~d} t \\
& \geq \sum_{\lambda_{j} \leq r}\left|\operatorname{Re} a_{j}\right|^{2} \int_{1}^{3} t^{2} \mathrm{~d} t=\frac{8}{3} \sum_{\lambda_{j} \leq r}\left|\operatorname{Re} a_{j}\right|^{2} .
\end{aligned}
$$


For the right term of (6.2), we have $\partial_{t} \operatorname{Re} y(x, 0)=\sum_{\lambda_{j} \leq r} \operatorname{Re} a_{j} e_{j}$ and

$$
|\operatorname{Re} y|_{H^{1}(Q)}^{2} \leq C \mathrm{e}^{8 \sqrt{r}}(1+r) \sum_{\lambda_{j} \leq r}\left|\operatorname{Re} a_{j}\right|^{2} \leq C \mathrm{e}^{9 \sqrt{r}} \sum_{\lambda_{j} \leq r}\left|\operatorname{Re} a_{j}\right|^{2} .
$$

Therefore we get

$$
\sum_{\lambda_{j} \leq r}\left|\operatorname{Re} a_{j}\right|^{2} \leq C\left(\int_{\omega}\left|\sum_{\lambda_{j} \leq r} \operatorname{Re} a_{j} e_{j}\right|^{2} \mathrm{~d} x\right)^{\mu \delta}\left(\mathrm{e}^{9 \sqrt{r}} \sum_{\lambda_{j} \leq r}\left|\operatorname{Re} a_{j}\right|^{2}\right)^{1-\mu \delta} .
$$

Hence we have

$$
\sum_{\lambda_{j} \leq r}\left|\operatorname{Re} a_{j}\right|^{2} \leq C \mathrm{e}^{C \sqrt{r}} \int_{\omega}\left|\sum_{\lambda_{i} \leq r} \operatorname{Re} a_{i} e_{i}\right|^{2} \mathrm{~d} x
$$

By the same argument, we can get

$$
\sum_{\lambda_{j} \leq r}\left|\operatorname{Im} a_{j}\right|^{2} \leq C \mathrm{e}^{C \sqrt{r}} \int_{\omega}\left|\sum_{\lambda_{i} \leq r} \operatorname{Im} a_{i} e_{i}\right|^{2} \mathrm{~d} x .
$$

From (6.7) and (6.8), we obtain

$$
\sum_{\lambda_{j} \leq r}\left|a_{j}\right|^{2} \leq C \mathrm{e}^{C \sqrt{r}} \int_{\omega}\left|\sum_{\lambda_{i} \leq r} a_{i} e_{i}\right|^{2} \mathrm{~d} x
$$

Acknowledgements. The author acknowledges the anonymous referees for valuable and helpful comments.

\section{REFERENCES}

[1] X. Fu, A weighted identity for partial differential operators of second order and its applications. C. R. Acad. Sci., Sér. I Paris 342 (2006) 579-584.

[2] A.V. Fursikov and O.Yu. Imanuvilov, Controllability of Evolution Equations, Lect. Notes Ser. Seoul National University, Seoul 34 (1996).

[3] E. Hebey, Nonlinear Analysis on Manifolds: Sobolev Spaces and Inequalities, Courant Lect. Notes Math. New York University Courant Institute of Mathematical Sciences, New York 5 (1999).

[4] D. Jerison and G. Lebeau, Nodal sets of sums of eigenfunctions, in Harmonic Analysis and Partial Differential Equations. Chicago, IL (1996) 223-239; Chicago Lect. Math., Univ. Chicago Press, Chicago, IL (1999).

[5] J. Jost, Riemann Geometry and Geometric Analysis. Springer-Verlag, Berlin, Heidelberg (2005).

[6] M.M. Larent'ev, V.G. Romanov and S.P. Shishat.Skii, Ill-posed Problems of Mathematical Physics and Analysis. Edited by Amer. Math. Soc. Providence. Transl. Math. Monogr. 64 (1986).

[7] G. Lebeau and L. Robbiano, Contrôle exact de l'équation de la chaleur. Commun. Partial Differ. Equ. 20 (1995) 335-356.

[8] G. Lebeau and L. Robbiano, Stabilizzation de l'équation des ondes par le bord. Duke Math. J. 86 (1997) $465-491$.

[9] G. Lebeau and E. Zuazua, Null controllability of a system of linear thermoelasticity. Arch. Ration. Mech. Anal. 141 (1998) 297-329.

[10] X. Liu and X. Zhang, On the local controllability of a class of multidimensional quasilinear parabolic equations. C. R. Math. Acad. Sci., Paris 347 (2009) 1379-1384.

[11] A. López, X. Zhang and E. Zuazua, Null controllability of the heat equation as singular limit of the exact controllability of dissipative wave equations. J. Math. Pure. Appl. 79 (2000) 741-808.

[12] Q. Lü, Bang-Bang principle of time optimal controls and null controllability of fractional order parabolic equations. Acta Math. Sin. 26 (2010) 2377-2386.

[13] Q. Lü, Control and Observation of Stochastic Partial Differential Equations. Ph.D. thesis, Sichuan University (2010). 
[14] Q. Lü, Some results on the controllability of forward stochastic heat equations with control on the drift. J. Funct. Anal. 260 (2011) 832-851.

[15] Q. Lü and G. Wang, On the existence of time optimal controls with constraints of the rectangular type for heat equations. SIAM J. Control Optim. 49 (2011) 1124-1149.

[16] L. Miller, How violent are fast controls for Schrödinger and plate vibrations? Arch. Ration. Mech. Anal. 172 (2004) $429-456$.

[17] J. Milnor, Morse Theory, Ann. Math. Studies. Princeton Univ. Press, Princeton, NJ (1963).

[18] K.-D. Phung and X. Zhang, Time reversal focusing of the initial state for Kirchhoff plate. SIAM J. Appl. Math. 68 (2008) $1535-1556$.

[19] G. Wang, $L^{\infty}$-null controllability for the heat equation and its consequences for the time optimal control problem. SIAM J. Control Optim. 47 (2008) 1701-1720.

[20] X. Zhang, Explicit observability estimate for the wave equation with lower order terms by means of Carleman inequalities. SIAM J. Control Optim. 39 (2001) 812-834.

[21] C. Zheng, Controllability of the time discrete heat equation. Asymptot. Anal. 59 (2008) 139-177.

$[22]$ E. Zuazua, Controllability and observability of partial differential equations: Some results and open problems, in Handbook of Differential Equations: Evolutionary Differential Equations 3 (2006) 527-621. 